\title{
Generation, Amplification, and Nonlinear Self-Compression of Powerful Superradiance Pulses
}

\author{
Naum S. Ginzburg, Irina V. Zotova, Adrian W. Cross, Alan D. R. Phelps, Member, IEEE, \\ Michael I. Yalandin, Member, IEEE, and Vladislav V. Rostov
}

\begin{abstract}
Superradiance (SR) of electron bunches can be considered an effective method of production of ultrashort electromagnetic pulses. Different types of SR associated with different mechanisms (cyclotron, Cherenkov, and bremsstrahlung) of stimulated emission are observed experimentally in the millimeter and centimeter wavelength bands. Progress in this research has enabled a new type of generator to be created capable of generating unique short (under 200-300 ps) electromagnetic pulses at super high peak powers exceeding $1 \mathrm{GW}$ in the millimeter and $3 \mathrm{GW}$ in the centimeter waveband. Some new methods for further increasing of the SR pulse peak power along with the promotion of such sources to higher frequency bands are discussed. These new methods include phase synchronization of several SR pulse generators, the amplification of an SR pulse during its propagation along a quasi-stationary electron beam and nonlinear compression in the process of induced selftransparency.
\end{abstract}

Index Terms-Bremsstrahlung, Cherenkov, cyclotron, free electron radiation, microwave, mm-wave, nonlinear selfcompression, terahertz sources, superradiance (SR), ultrashort pulses.

\section{INTRODUCTION}

$\mathbf{E}$ FFECTS arising from the generation, amplification, and propagation of short (in the scale of relaxation times) light pulses in two-level inverted (active) and noninverted (passive) resonance media are well known in optics and were studied in detail theoretically and experimentally [1]-[6], [8]. The best known effects of this class are superradiance (SR) [1]-[3], self-induced transparency (SIT) [4]-[6] and nonlinear amplification with simultaneous compression [7], [8]. It is obvious that similar effects can occur in the interaction of electromagnetic radiation with beams and bunches of classical electrons.

Manuscript received December 16, 2012; revised February 28, 2013; accepted March 4, 2013. Date of publication April 6, 2013; date of current version April 6, 2013.

N. S. Ginzburg and I. V. Zotova are with the Institute of Applied Physics RAS, N. Novgorod 603950, Russia (e-mail: ginzburg@ appl.sci-nnov.ru; zotova@appl.sci-nnov.ru).

A. W. Cross and A. D. R. Phelps are with the Department of Physics, SUPA, University of Strathclyde, Glasgow G4 0NG, U.K. (e-mail: a.w.cross@strath.ac.uk; a.d.r.phelps@strath.ac.uk).

M. I. Yalandin is with the Institute of Electrophysics RAS, Ekaterinburg 620016, Russia (e-mail: yalandin@iep.uran.ru).

V. V. Rostov is with the Institute of High Current Electronics RAS, Tomsk 634055, Russia (e-mail: rostov@1fe.hcei.tsc.ru).

Color versions of one or more of the figures in this paper are available online at http://ieeexplore.ieee.org.

Digital Object Identifier 10.1109/TPS.2013.2252369
Recently, significant progress is achieved in the generation of powerful ultrashort microwave pulses based on the SR of extended electron bunches. Coherent emission from the entire volume of such bunches can occur because of self-bunching and slippage of the wave over the electron pulse [9]-[12]. The SR of classical electrons can be associated with different mechanisms of stimulated emission, such as cyclotron, Cherenkov, and bremsstrahlung. All the above types of SR are observed experimentally at millimeter and centimeter wavelength bands [13]-[25]. Currently, the SR pulses with the highest peak power are obtained for the Cherenkov mechanism of SR when the electrons interact with synchronous harmonics of the backward wave propagating in slow wave structures (SWSs) [17]-[25]. Progress in this research has enabled a new type of generator to be created capable of generating unique short (under 200-300 ps) electromagnetic pulses at super high peak powers exceeding $1 \mathrm{GW}$ in the millimeter and $3 \mathrm{GW}$ in the centimeter wavebands.

This review paper has the following organization. Section I describes the theoretical and experimental studies of Cherenkov mechanisms of SR in the process of interaction of an electron bunch with a backward wave. Recent experiments with phase synchronization of several SR pulse generators [26]-[32] are also discussed. Section II describes the simulation and experimental observation of amplification of SR pulses during propagation along quasi-stationary electron beams [33], [34]. Section III describes an alternative method for further increasing the SR pulse peak power associated with nonlinear compression in the process of induced selftransparency [35], [36]. Section IV describes the development of SR sources for higher frequency bands.

\section{Cherenkov SR}

\section{A. Basic Model and Simulations}

The Cherenkov SR pulses can be produced by a bunch of electrons moving along rectilinear trajectories in SWSs. The SR pulses of maximal power are generated in interaction with the slow spatial harmonic of the backward wave in periodically corrugated waveguide. According to the Floquet theorem, the electric field in the periodically corrugated waveguide can be presented as a sum of spatial harmonics

$$
\vec{E}(z, t)=\operatorname{Re}\left(A(z, t) \sum_{-\infty}^{+\infty} \overrightarrow{\tilde{E}}^{(n)}\left(\vec{r}_{\perp}\right) e^{i \omega t+i n \bar{h} z+i h z}\right)
$$


where $\omega$ is the carrier frequency, $h$ is the longitudinal wavenumber of the fundamental harmonic, $\bar{h}=2 \pi / d, d$ is the period of the corrugation, $A(z, t)$ is the slowly varying complex wave amplitude, and $\overrightarrow{\tilde{E}}^{(n)}$ is the transverse field profile of the spatial harmonics. When the parameters of the corrugation (period or depth) are slightly tapered along the interaction space the amplitudes of the harmonics $\overrightarrow{\tilde{E}}^{(n)}$ should be slow functions of the longitudinal coordinate $z$. Assuming the magnetized electrons move in the axial direction only and interact with the single slow spatial harmonic $(n=-1)$ of the backward wave under the synchronism condition

$$
\omega=(-h+\bar{h}) v_{\| 0}
$$

where $v_{\| 0}=\beta_{\| 0} c$ is the unperturbed longitudinal velocity of the electrons. Due to a small variation of the particles' velocity, the radiation of the electron bunch can be described by the following:

$$
\begin{aligned}
\frac{\partial a}{\partial \tau}-\frac{\partial a}{\partial Z} & =\chi(Z) f(\tau) J \\
\frac{\partial^{2} \theta}{\partial Z^{2}} & =\left[1+2 \gamma_{0}^{2} C \frac{\partial \theta}{\partial Z}\right]^{3 / 2} \chi(Z) \operatorname{Re}[a \exp (i \theta)] .
\end{aligned}
$$

Here, we use the following dimensionless variables:

$$
\tau=\frac{\omega C\left(t-z / v_{\| 0}\right)}{1+v_{\| 0} / v_{\mathrm{gr}}}, \quad Z=\frac{\omega C z}{v_{\| 0}}, \quad a=\frac{e A\left\langle E_{\perp}\left(r_{b}, z\right)\right\rangle}{C^{2} \gamma_{0}^{3} v_{\| 0} m \omega}
$$

where $\theta=\omega\left(t-z / v_{\| 0}\right)$ is the phase of an individual particle with respect to the field of the synchronous harmonic, $v_{\mathrm{gr}}$ is the group velocity of the electromagnetic wave, $\gamma_{0}=$ $\left(1-\beta_{\| 0}\right)^{-1 / 2}, C=\left(e I_{b} \widehat{Z} / 2 m c^{2} \gamma_{0}^{3}\right)^{1 / 3}$ is the Pierce parameter, $\widehat{Z} \equiv 2\left\langle\tilde{E}_{z}^{(-1)}\left(r_{b}\right)\right\rangle^{2} / k^{2} N$ is the mean (along the interaction distance) coupling impedance of the tapered SWS with the full length $l,\left\langle\tilde{E}_{z}^{(-1)}\left(r_{b}\right)\right\rangle=(1 / l) \int_{0}^{l} \tilde{E}_{z}^{(-1)}\left(r_{b}, z\right) d z, k=\omega / c, N$ is the waveguide norm, $I_{b}$ is the electron current, and $r_{b}$ is the electron injection radius. The function $\chi(Z)$ describes the longitudinal profile of the wave coupling impedance (that can be tapered, for e.g., by varying the corrugation depth). This function is normalized as $(1 / L) \int_{0}^{L} \chi(Z) d Z=1$, where $L=\omega C l / v_{\| 0}$ is the dimensionless length of the interaction space. The function $f(\tau)$ describes the current pulse profile. We assume that electron density is constant $(f(\tau)=1, \tau \in$ $[0, T])$ within the dimensionless bunch duration as follows:

$$
T=\frac{\omega C}{1+v_{\| 0} / v_{\mathrm{gr}}} \Delta t_{b} .
$$

Apart from small initial density perturbations at the system entrance

$$
\begin{aligned}
& \left.\theta\right|_{Z=0}=\theta_{0}+\tilde{J} \cos \theta_{0}, \quad \tilde{J} \ll 1 \\
& \theta_{0} \in[0,2 \pi), \quad \partial \theta /\left.\partial Z\right|_{Z=0}=0 .
\end{aligned}
$$

These perturbations initiate the development of SR emission.

For uniform SWS $[\chi(Z)=1]$ formation of a short SR pulse is shown in Fig. 1(a). It is found from solution to the differential (3). This process is caused by electron bunching and slippage of the wave with respect to the electrons because of the difference between the wave group velocity and the electron longitudinal velocity. The distinctive feature of SR

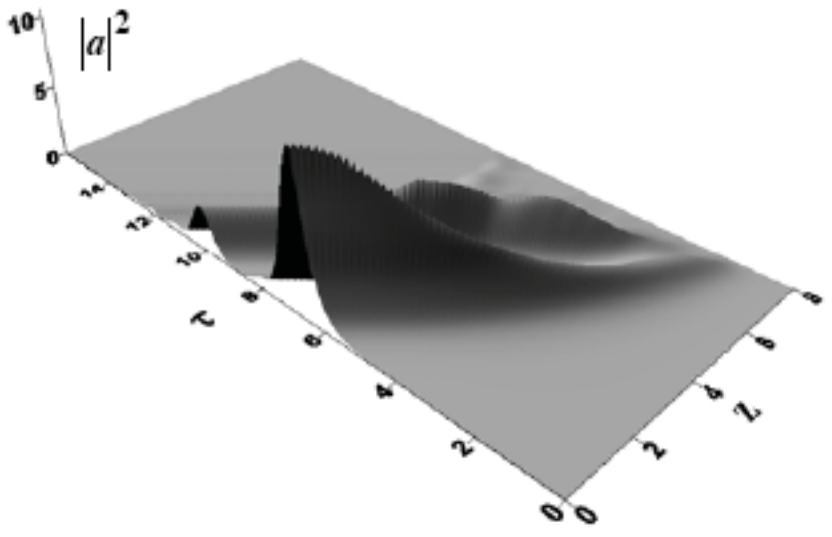

(a)

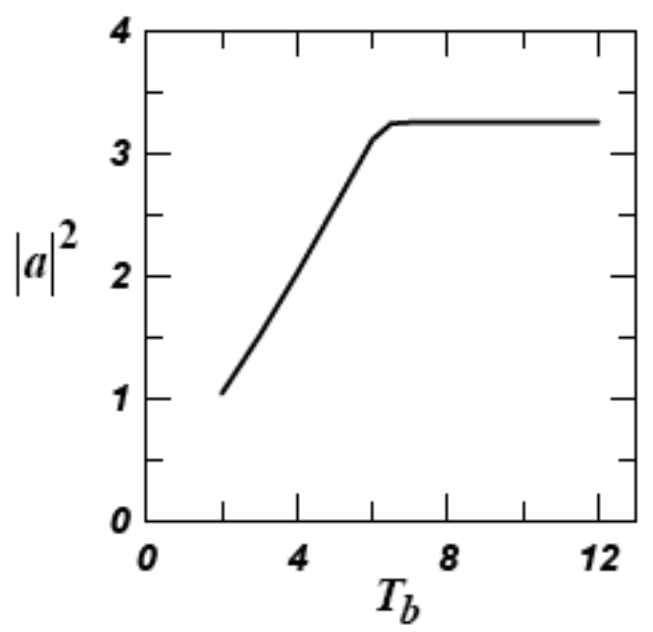

(b)

Fig. 1. (a) Formation of short SR pulse by electron bunch moving in uniform SWS ( $\left.L=8, T_{b}=8, C=0.02, \tilde{J}=0.01\right)$. (b) Dependence of SR pulse peak amplitude on electron pulse duration for fixed beam current. [Solutions of differential (3) for $\chi(Z)=1$.]

is the linear dependence of peak power on the square of the number of radiating particles [1]-[3]. The dependence of the SR pulse amplitude on the electron pulse duration is shown in Fig. 1(b) for constant beam current. The peak amplitude is proportional to the electron pulse duration until this duration is rather short $T<6$. It corresponds to a square law dependence of the radiation power on the total number of electrons in the bunch. Saturation of peak amplitude growth occurs when the electron pulse duration exceeds a certain value $T>6$, and the electron pulse becomes too long to provide coherent radiation from all particles over the pulse length. For a rather long electron bunch, the multispikes generation regime is realized, because the different parts of a bunch radiate practically independently. The backward wave interaction such a regime is similar to the self-modulation regime in a back wave oscillator (BWO) driven by a quasi-stationary electron beam [37].

In certain conditions, the SR pulse peak power can substantially exceed the power of the electron beam because a short SR pulse that forms in the initial stage of the interaction can accumulate energy from different fractions of the extended 


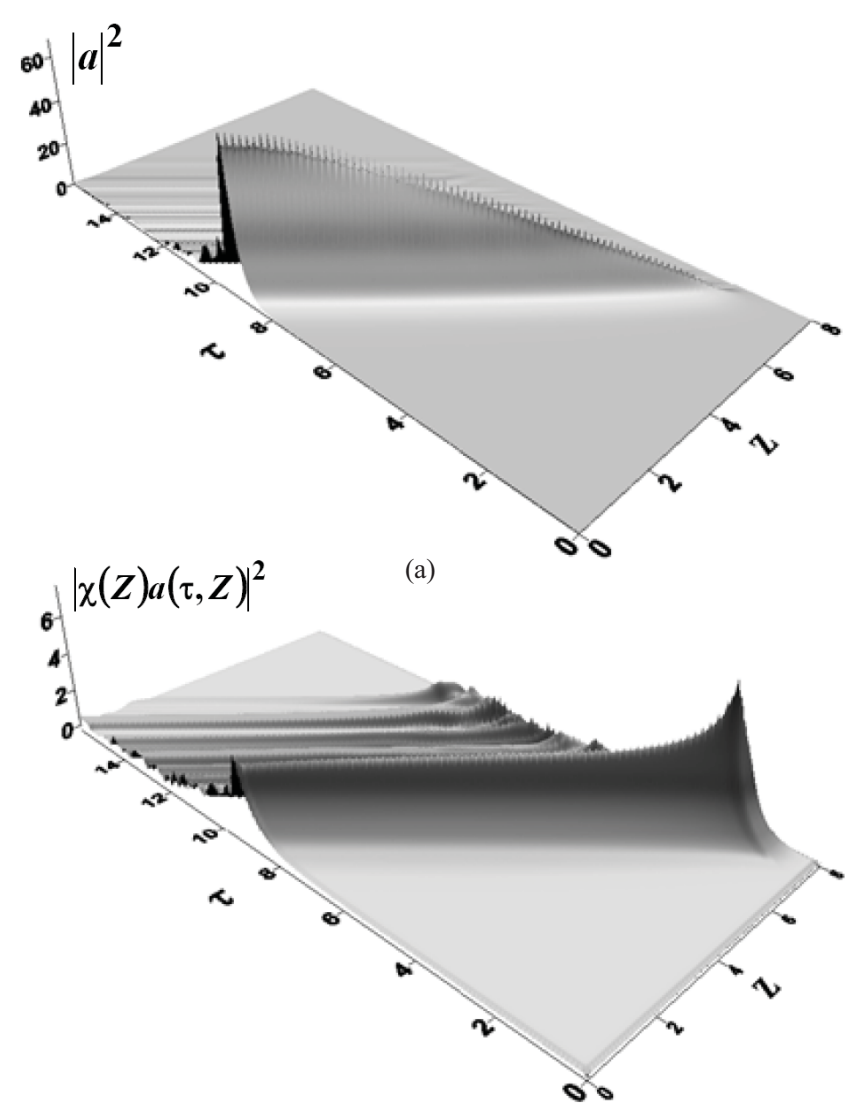

(b)

Fig. 2. (a) Formation of SR pulse in optimized nonuniform SWS with peak power exceeding electron bunch power $(K>4)$. (b) Amplitude of synchronous space harmonic. [Solutions of differential (3) for $\chi(Z)$ given by (7)].

electron bunch. This fact does not contradict the energy conservation law because the SR pulse total energy is still smaller than the total beam kinetic energy. Let us introduce the power conversion factor as the ratio of the SR pulse peak power to the electron beam power

$$
K=\gamma_{0}^{2} C|a|_{\max }^{2} \frac{\left(\gamma_{0}+1\right)}{4} .
$$

In a uniform SWS, the conversion factor $K<1$ for ultrarelativistic energy of electrons $\left(\gamma_{0} \gg 1\right)$. In the range of moderate electron energies $\sim 300-500 \mathrm{keV}$ the conversion factor can slightly exceed unity $K \sim 1.2$ and $K$ reaches a maximum value $\sim 1.4$ for nonrelativistic energies [21].

To obtain a substantial excess of the power conversion factor over unity, it is necessary to use the nonuniform SWS with variable coupling impedance. It is beneficial to realize a situation where despite the linearly growing energy and power of the microwave pulse, as it propagates through the system, the electric field strength in the synchronous harmonic acting upon the electrons is a constant. The impedance profile satisfying the above condition may be presented in the form [21]

$$
\chi(\zeta)= \begin{cases}\frac{a_{0}}{\left[p_{0}-\left(p_{0}-1\right) \frac{Z}{Z_{0}}\right]^{1 / 2}}, & \text { if } 0 \leq Z \leq Z_{0} \\ a_{0}, & \text { if } Z_{0} \leq Z \leq L\end{cases}
$$

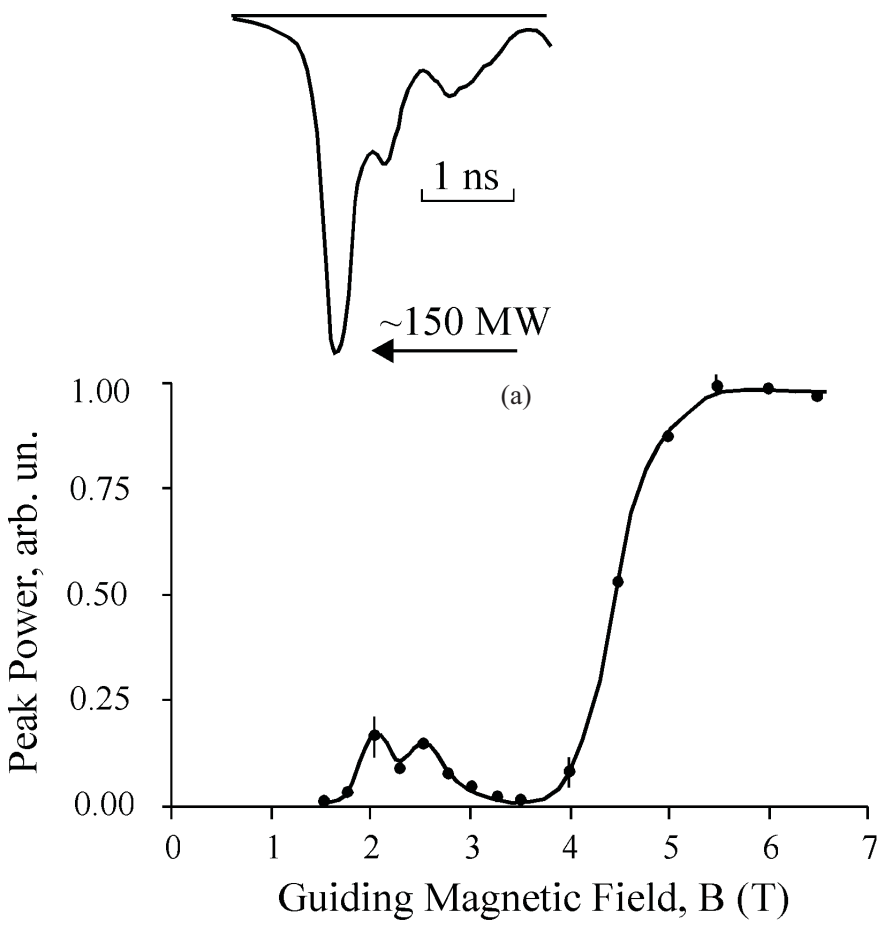

(b)

Fig. 3. Ka-band. (a) SR pulse power as a function of time. (b) Dependence of SR pulse peak power on value of guide magnetic field.

where the parameter $p_{0}$ characterizes the relative impedance variation. The evolution of the radiation power $|a(\tau, Z)|^{2}$ in the case of a tapered coupling impedance for $p_{0}=90$ and $Z_{0}=0.93 L$ are shown in Fig. 2(a). In this situation, the amplitude of the synchronous space harmonic $|\chi(Z) a(\tau, Z)|$ is practically constant [see Fig. 2(b)]. In this considered situation, a conversion factor $K$ of 4 may be achieved. Thus, the use of a nonuniform profile allows the SR pulse peak power to be increased significantly.

\section{B. Experimental Observation of Cherenkov $S R$ in Uniform Slow Wave Structures}

The results of the theoretical analysis are confirmed by the experiments in millimeter and centimeter wavebands based on high-current accelerators.

At Ka-band the experiments are performed based on the RADAN-303BP accelerator, which can provide $1 \mathrm{~ns}$, $270-290-\mathrm{kV}$ accelerating voltage pulses. In the first experiments, a uniform SWS in the form of a periodically corrugated waveguide is used with corrugation period $0.35 \mathrm{~cm}$, depth $0.075 \mathrm{~cm}$, and mean diameter $D / \lambda=0.8$ [17], [18]. Highcurrent electron bunches are transported through the interaction space in a longitudinal guiding magnetic field created by a pulsed solenoid ( $B \sim 2.5-5 \mathrm{~T}$ ) or by a superconducting magnet $\left(B_{\max } \sim 8.5 \mathrm{~T}\right)$. Similar to the traditional BWO, the fall in the power near the magnetic field value $\sim 3 \mathrm{~T}$ is caused by cyclotron absorption when the cyclotron resonance condition for the fundamental harmonic is matched [Fig. 3(b)]. The maximal peak power of the SR pulse is obtained for a value of the magnetic field exceeding the cyclotron resonance 
value. A typical oscilloscope trace of the Ka-band microwave signal is shown in Fig. 3(a). The observed microwave pulses had a duration of $\sim 300 \mathrm{ps}$ and a rise time of $200 \mathrm{ps}$. Frequency measurements using a set of cutoff waveguide filters showed that the main peak had a central frequency $\sim 38 \mathrm{GHz}$ with relative radiation spectrum bandwidth of $\sim 5 \%$. The radiation pattern corresponded to the excitation of the $\mathrm{TM}_{01}$ mode. The absolute peak power is calculated by integrating the signal from the detector over its radial position. For the electron bunch with energy of $\sim 290 \mathrm{keV}$ and peak current of $\sim 2 \mathrm{kA}$ the peak power is $\sim 150 \mathrm{MW}$ in the single pulse regime. For the same SWS operation in the repetition rate mode at $25 \mathrm{~Hz}$ is realized with a peak power of the SR pulses $\sim 60 \mathrm{MW}$.

Alongside the investigation of the generation of subnanosecond SR pulses at Ka-band, similar experiments are performed at W-band $(75 \mathrm{GHz})$ and G-band $(150 \mathrm{GHz})$ [19]. The Wband SR pulses possessed a sharp leading edge $<120 \mathrm{ps}$ and peak power $\sim 10-15 \mathrm{MW}$. In the G-band, the measured pulse rise time did not exceed 75 ps which is the limit of the transient characteristic of the 5-GHz oscilloscope. The estimated W-band SR pulse peak power is not $<5-10 \mathrm{MW}$.

To increase the SR pulse peak power in the following experiments in Ka-band, an advanced SWS is used [20]. Obviously, an important factor limiting the peak power is dispersion spread of the SR pulse during its propagation through the SWS in the backward and forward directions. To diminish the influence of this factor, a slightly oversized SWS with $D / \lambda=1.3$ and a resonant reflector for electromagnetic pulse extraction are implemented. For electron bunches with current $2.1 \mathrm{kA}$ and a particle energy of $290 \mathrm{keV}$ in a highmagnetic field (5.5 T), using the new advanced SWS allowed 200-250 ps microwave pulses to be obtained with a maximum peak power of $420 \mathrm{MW}$. In a low magnetic field $(\sim 2 \mathrm{~T})$ the peak power decreased to 240-280 MW.

\section{SR Experiments With Nonuniform Slow Wave System. Pro- duction of SR Pulses With Peak Power Exceeding the Electron Beam Power}

The next step is the use of nonuniform SWS with coupling impedance that increased toward the collector end [21]-[25]. The first experiments using nonuniform SWS are performed in the X-band [21]. As a source of electrons, the accelerator based on the SINUS-150 compact generator is used. It provided a 4-ns electron bunch with current of $2.6 \mathrm{kA}$ and particle energy of $330 \mathrm{keV}$. The SWS had a length of $L=36 \mathrm{~cm}(\sim 12 \lambda)$ with a corrugation period of $1.3 \mathrm{~cm}$. Thus, the maximal power of the electron bunch achieved $0.85 \mathrm{GW}$. The strength of the magnetic field, generated by a pulsed solenoid, reached $3 \mathrm{~T}$. The pulse energy achieved is $\sim 0.6 \mathrm{~J}$. The power conversion coefficient is estimated to be 1.4 with a maximum microwave power of $1.2 \mathrm{GW}$.

The next experiment with maximum power conversion achievement $(K \sim 1.8)$ is performed by using the SINUS-200 compact generator (pulse width $9 \mathrm{~ns}$, voltage up to $330 \mathrm{kV}$, and beam current $5 \mathrm{kA}$ ). The SWS had the length of $L=65 \mathrm{~cm}$ $(\sim 20 \lambda)$. The amplitude of the corrugation increased from 0.1 to $0.25 \mathrm{~cm}$ along the system (that corresponded to a

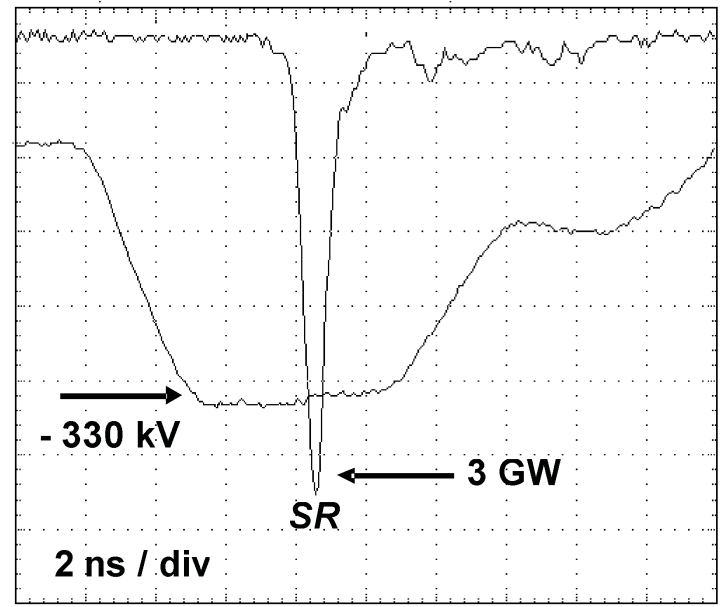

Fig. 4. Waveforms of vacuum diode voltage and microwave detector signal for X-band SR in nonuniform SWS.

variation of the coupling impedance from $\sim 0.3$ up to $\sim 2 \Omega$ ). The waveforms of the vacuum diode voltage and microwave detector signal are shown in Fig. 4. After reconstruction of the signal shape considering the nonlinearity of the detector, the following parameters of the microwave pulse are obtained: central frequency $9.3 \mathrm{GHz}$ and peak power $\sim 3 \mathrm{GW}$ with a 0.65-ns width.

Similar experiments are carried out in Ka-band [22] based on the RADAN 303BP accelerator. The nonuniform SWS with increasing corrugation depth had a length of $\sim 12 \mathrm{~cm}(\sim 14 \lambda)$ and a mean diameter $\sim 1.3 \lambda$. The SWS is optimized numerically using the axially symmetric version of the KARAT code. The simulation included the formation of the electron bunch in the coaxial vacuum diode. The geometry of the experimental setup used in the simulation is presented in [22]. In the simulation, it is demonstrated that in a strong magnetic field of up to $6.5 \mathrm{~T}$ it is possible to achieve a conversion factor of $\sim 1.7$. Thus, a $2.5 \mathrm{kA}, 290-\mathrm{keV}$ electron bunch with a power $\sim 700 \mathrm{MW}$ can emit a SR pulse with a peak power of over $1 \mathrm{GW}$ and duration $<300$ ps (Fig. 5).

The particle-in-cell (PIC)-simulations demonstrated that in the low guiding magnetic field $\sim 2 \mathrm{~T}$ (less than the cyclotron resonance value) the peak power of the SR pulse generated in a nonuniform SWS should be $\sim 400 \mathrm{MW}$. This regime is used in the experiment for production of SR pulses at a highrepetition rate [23]. These experiments are performed in the Ka-band exploiting a hybrid high-voltage modulator consisting of a SM-3NS nanosecond driver and a subnanosecond hydrogen peaking switch. The driver had several stages of energy compression based on solid-state opening switches. The experimental setup is shown in Fig. 6 together with a typical microwave signal recorded by the 6-GHz stroboscopic oscilloscope. This waveform is obtained for a $1-\mathrm{kHz}$ pulse repetition frequency. Processing the detector signal yielded a pulse width equal to 250 ps with a 190-ps rise time. The microwave energy measured by a calorimeteric method is 65-mJ per pulse, which corresponds to a peak power of 260 MW. The average microwave power over the train of pulses is $200 \mathrm{~W}$ at a pulse repetition frequency of $3.5 \mathrm{kHz}$. In a 


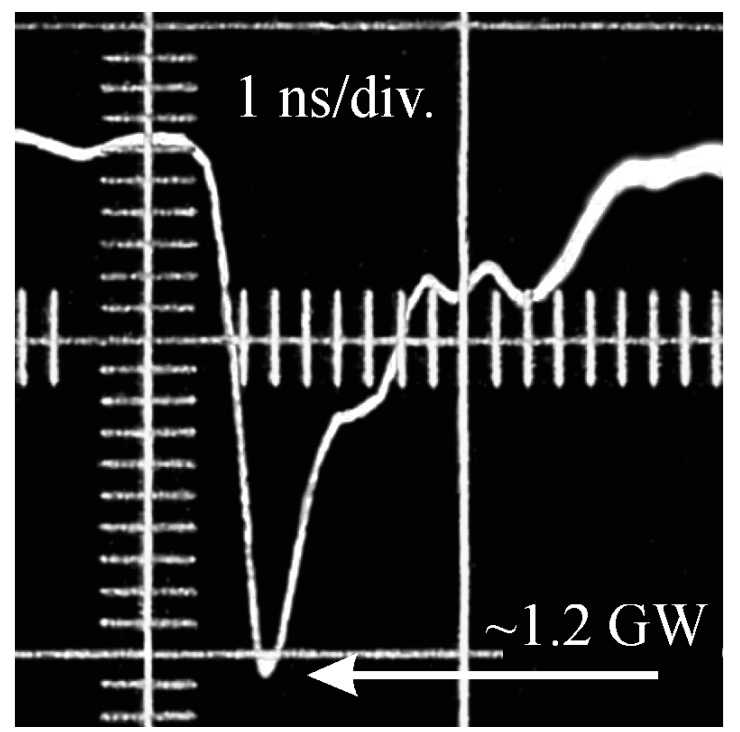

Fig. 5. Oscilloscope trace of 1-GW 300 ps (full-width at half-maximum) Ka-band SR pulse.

few series of experiments with the electron beam parameters increased to the limit allowed by the modulator, microwave pulses with $80 \mathrm{~mJ}$ of energy and $300 \mathrm{MW}$ of peak power are produced.

\section{Coherent Summation of SR Pulses Emitted from Several Channels}

S-band [26] and X-band [27] SR experiments demonstrated high stability of pulse-to-pulse phase distribution of the radiation. The root-mean-square (RMS) phase deviation on the time scale is found to approximate the rise time dispersion of the supply voltage. A fundamentally important problem that should be solved for coherent summation of SR pulses emitted from several channels is the stabilization of the explosive electron emission (EEE) delay, which is generally variable in a wide range in response to slightly varying field strength at the edge of a cylindrical cathode. The EEE delay does not destabilize the microwave pulse phase pattern; its dispersion should be restricted in the range from several to $10 \mathrm{ps}$. This requires a fast rise time high-voltage pulse to be applied to the cathode. The best suited cathodes are dense graphite cathodes with an extremely thin edge of $\sim 0.1-0.3 \mathrm{~mm}$ as well as metal-dielectric (MD) film cathodes. Particularly, an increase in RMS EEE delay from 2-3 to 8 ps in $10^{4}$ pulses is observed in experiments with two-channel injection of parallel beams from identical graphite cathodes [28]. In a special experiment, the lifetime of MD cathodes is found to be even better. The EEE delay did not increase noticeably in $\sim 10^{6}$ pulses.

The results [26]-[28] let rise to investigations of advanced HPM systems like phased antenna arrays on the basis of X-band SR BWOs without electrodynamic coupling [29], [30]. As in [31], SR BWOs are capable of operating at high-pulse repetition rates and demonstrating stability of both microwave pulse amplitude and shape. The idea of phase synchronization between two channels can be realized if the sharpest parts
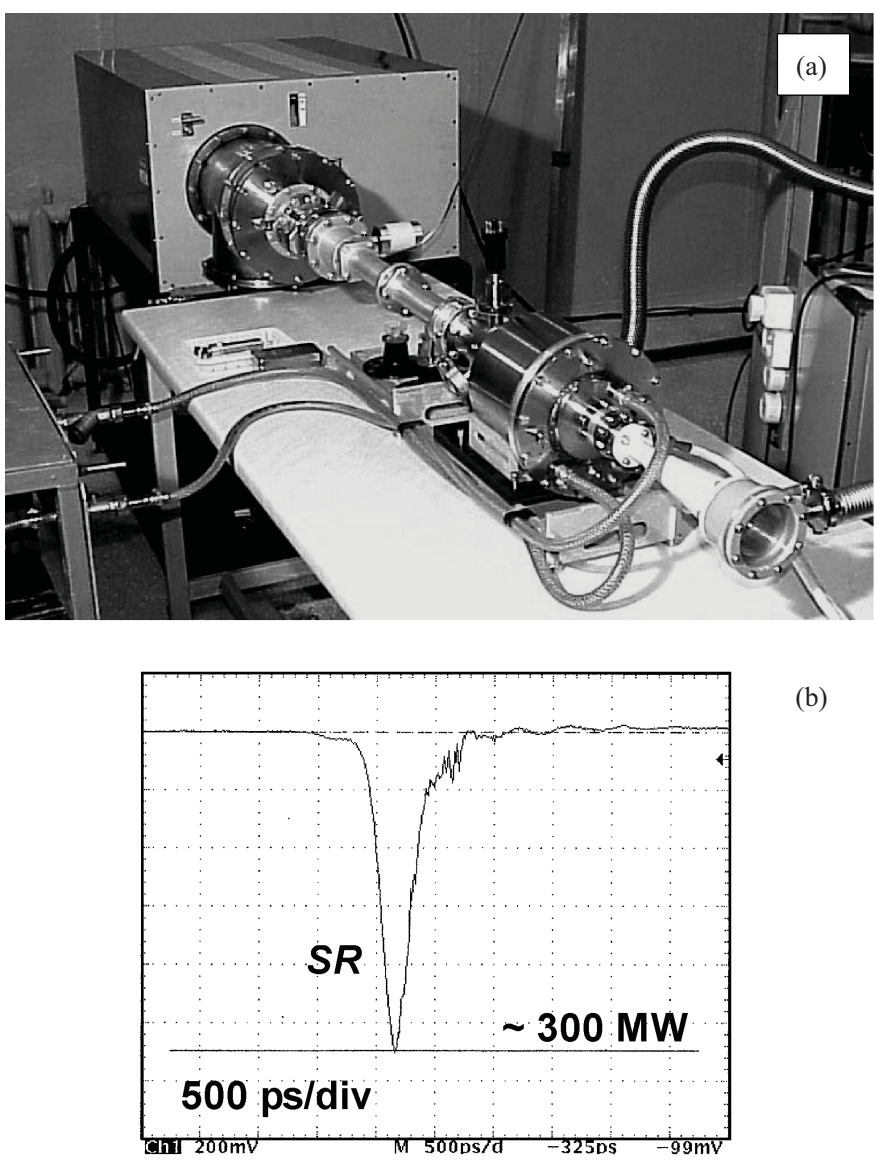

(b)

Fig. 6. Experimental setup for production of Ka-band SR pulses with repetition frequency $3.5-\mathrm{kHz}$ based on hybrid modulator and typical microwave signal recorded by 6-GHz stroboscopic oscilloscope.

of identical current fronts of the beams arrive at the input of the interaction space with a negligible time jitter. If so, then precisely-adjustable time shift between the current fronts provides controllable tuning of the phase difference for two microwave pulses [30].

For experiments [29], [30], a laboratory setup (Fig. 7) based on a SINUS-200 high-voltage accelerator with a pulse forming line (PFL) of impedance $30 \Omega$ and electrical length $9 \mathrm{~ns}$ is used. The accelerator allowed repetitive pulsed operation at $100 \mathrm{~Hz}$. In the self-breakdown mode, the main gas gap switch ensured TEM voltage pulses of amplitude up to $300 \mathrm{kV}$ in a coaxial transmission line [Fig. 8(a)]. The electrical length of the transmission lines downstream of a coaxial T-branch ensured reliable decoupling between the channels. The vacuum diodes and the SWSs of the SR BWO are identical in geometry and are placed inside the solenoids (B-field of $\sim 0.5 \mathrm{~T}$ ) connected in series to the dc supply. In the first stage of the experiments [29], a single peaking switch (peaker) based on a nonlinear line with an unsaturated ferrite section is located at the output of the PFL, as shown in Fig. 8. Stable operation of the gas gap switch and the solid-state sharpener provided the conditions for mutual phasing of two X-band SR BWOs with an accuracy $\sigma \leq 2 \%$. Thus, coherent summation of the radiated fields of independent generators is shown for the first time. The relative duration of $300-\mathrm{MW}$ 


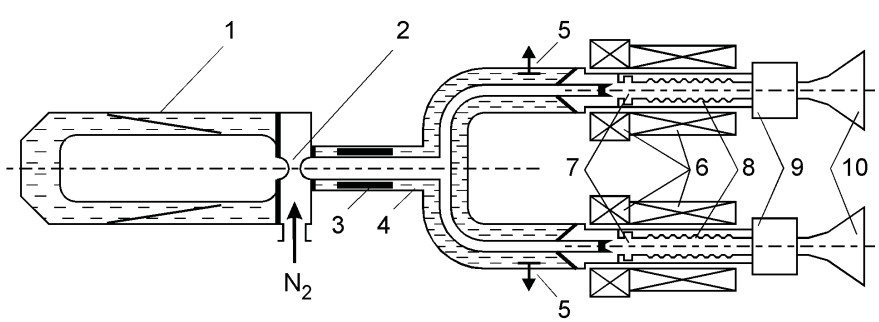

Fig. 7. Experimental device with parallel SR BWOs: 1: SINUS-200 highvoltage pulse generator. 2: Spark gap switch with gas blowing. 3: Ferrite peaker. 4: Coaxial transmission lines. 5: Capacitive voltage dividers. 6: Windings of dc solenoids. 7: Resonant reflectors. 8: SWSs. 9: Converters of $\mathrm{TM}_{01}$ wave to $\mathrm{TE}_{11}$ wave of circular waveguide. 10: Horn antennas with vacuum windows.
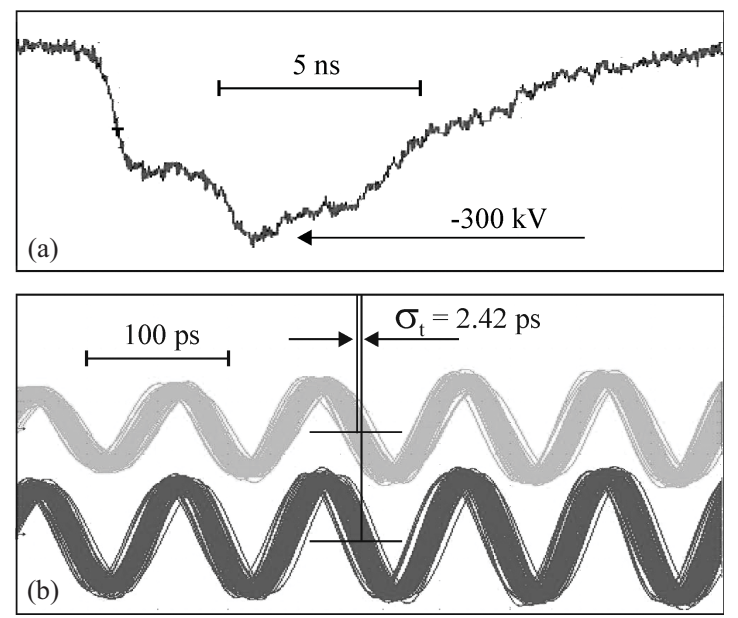

Fig. 8. Waveforms of (a) voltage pulse applied to cathodes and (b) set of 100 $\mathrm{X}$-band FR signals at a pulse repetition rate of $50 \mathrm{~Hz}$ in two channels produced for the measurements of the standard deviation in phase difference of the microwave oscillations $\left(\sigma_{t}=2.2 \mathrm{ps}\right)$.

SR pulses is $\left(T_{\text {pulse }} / T_{X}\right) \approx 20$, where $T_{\text {pulse }}$ is the microwave pulse duration and $T_{X}=100 \mathrm{ps}$ is the period of the carrier frequency.

In the second stage of the experiments [30], two ferritebased peakers positioned in the individual channels between the T-branch and the vacuum diodes are used. Both ferriteloaded nonlinear lines possessed controllable sharpening because of the variable ferrite saturation in the adjustable axial magnetic field created by the dc solenoids. The maximum rate rise of the voltage attained is $430 \mathrm{kV} / \mathrm{ns}$. The shift of the sharpened pulse front (by $\sim 100 \mathrm{ps}$ per $3 \mathrm{kA} / \mathrm{m}$ ) allowed a controllable relative phase shift of the radiation in the channels within more than the period $T_{X}$. Characteristic dispersion of the phase difference is kept at a level of several picoseconds [29]. This provided experimental demonstration of superradiative directional pattern scanning in free space. It is pertinent that phase stabilization and RF pattern scanning for the two-channel X-band device stimulated further similar researches in the Ka-band, where both coherent power summation and pattern scanning are demonstrated todate for the elongated pulsewidth of $\left(T_{\text {pulse }} / T_{K a}\right) \approx 150$ [32].

\section{Amplification And Nonlinear COMPRESSion of Ultrashort MicrowaVe PUlses by QUASI-STATIONARY ELECTRON BEAMS: THEORY AND EXPERIMENT}

For peak power enhancement of SR pulses, it is possible to use the subsequent amplification of a pulse during its propagation along a quasi-stationary electron beam. As theoretically shown in [33] and [34], the amplification of such short pulses is significantly different from the well-known mechanism for amplification of monochromatic signals in the $\mathrm{CW}$ regime. For short pulses essential effects are caused by slippage of the input pulse over the electron beam because of a difference of the radiation group velocity $v_{\mathrm{gr}}$ and the translational velocity of the particles $v_{\| 0}$. Under such conditions the leading, or tail, pulse fronts (depending on the ratio between $v_{\mathrm{gr}}$ and $v_{\| 0}$ ) are permanently fed by fresh electrons without coherent density modulation. Therefore, the input pulse accumulates energy from different fractions of the electron beam and is effectively amplified. After some propagation distance, the peak power of the SR pulse can exceed the power of the electron beam. Simultaneously, electromagnetic pulse shortening is observed. Results of theoretical analyses and the first experimental observations of amplification and nonlinear compression of SR pulses are presented. The Cherenkov mechanism of interaction in a dielectric loaded waveguide is used. For comparison with the experiments, the theoretical consideration of the above process is carried out.

\section{A. Basic Model and Simulations}

The process of amplification of a short electromagnetic pulse

$$
E_{z}=\operatorname{Re}\left\{E\left(\vec{r}_{\perp}\right) A(z, t) \exp (i \omega t-i h z)\right\}
$$

by a quasi-continuous electron beam in a dielectric loaded waveguide under the Cherenkov synchronism condition

$$
\omega \approx h v_{\| 0}
$$

can be described by the following equations [compare with (3)]:

$$
\begin{aligned}
\frac{\partial a}{\partial \tau}+\frac{\partial a}{\partial Z} & =f(\tau) J \\
\frac{\partial^{2} \theta}{\partial Z^{2}} & =\left[1+2 \gamma_{0}^{2} C \frac{\partial \theta}{\partial Z}\right]^{3 / 2} \operatorname{Re}[a \exp (i \theta)]
\end{aligned}
$$

with the boundary conditions

$$
\begin{aligned}
& \left.a\right|_{Z=0}=a_{0}(\tau),\left.\quad \theta\right|_{Z=0}=\theta_{0}+\tilde{J} \cos \left(\theta_{0}+\varphi(\tau)\right) \\
& \theta_{0} \in[0,2 \pi], \quad \partial \theta /\left.\partial Z\right|_{Z=0}=0 .
\end{aligned}
$$

Here

$$
\tau=\frac{\omega C\left(t-z / v_{\| 0}\right)}{1-v_{\| 0} / v_{\mathrm{gr}}}, \quad Z=\frac{\omega C z}{v_{\| 0}}, \quad a=\frac{e A E\left(r_{b}\right)}{C^{2} \gamma_{0}^{3} v_{\| 0} m \omega} .
$$

For the interaction with a $T M_{01}$ mode of a dielectric loaded waveguide $E=I_{0}\left(\kappa_{\perp} r_{b}\right)$, where $\kappa_{\perp}$ is the transverse 


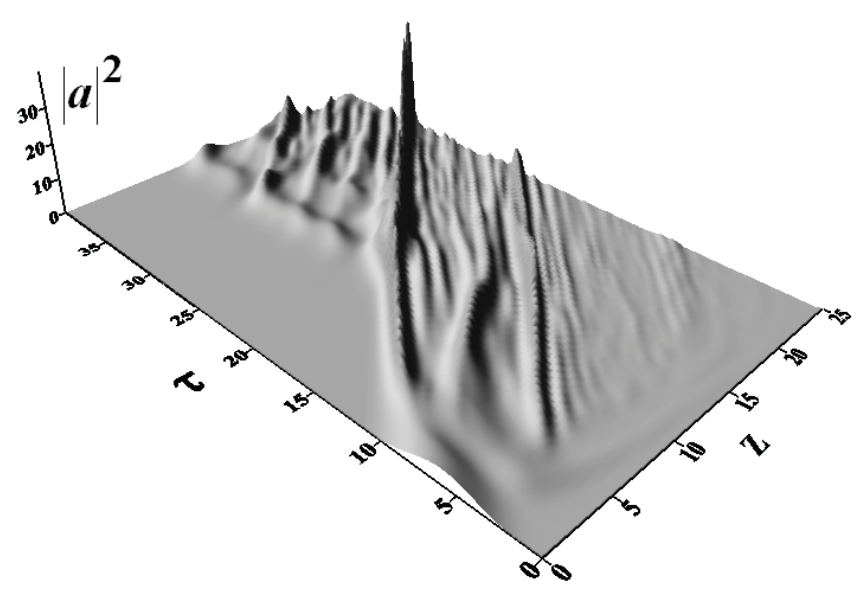

Fig. 9. Evolution of input SR pulse amplified by continues electron beam. $\left(L=25, a_{0}=2, T=12, C=0.1\right.$, and $\left.r=0.007\right)$.

wavenumber in the vacuum channel and $I_{0}(x)$ is the modified Bessel function. The gain parameter can be written as

$$
C=\left(\frac{e I_{b}}{m \omega_{s}^{2} \gamma_{0}^{3}} \frac{p^{4} I_{0}^{2}\left(\kappa_{\perp} R_{0}\right)}{N_{s}}\right)^{1 / 3}
$$

where $N_{s}$ is the norm of the operating mode (see [33]). The function $a(\tau)=a_{0} \sin ^{2}(\pi \tau / T)$ describes the form of the input signal with amplitude $a_{0}$ and duration $T$. The parameter $\tilde{J} \ll 1$ is introduced to consider the influence of noise modulation with random phase $\varphi(\tau)$.

Solutions of (10) are carried out for the parameters corresponding to the experimental values. The interaction space represented the cylindrical waveguide with radius $R=0.45 \mathrm{~cm}$ and length $24 \mathrm{~cm}$ with a dielectric quartz $(\varepsilon \sim 3.7)$ insert and width $0.135 \mathrm{~cm}$. For a particle energy of $300 \mathrm{keV}\left(\gamma_{0}=1.6\right)$, beam current $1 \mathrm{kA}$, and injection radius $R_{0}=0.2 \mathrm{~cm}$, the gain parameter $C \sim 0.1$. Correspondingly, the normalized interaction length is $L \sim 25$. The evolution of the input pulse with amplitude $a_{0}=2$ and duration $T=12$, which corresponds to a 300-ps incident signal with peak power $\sim 50 \mathrm{MW}$, is shown in Fig. 9.

The level of noise modulation of the electron density is chosen as $\tilde{J}=0.007$ that corresponds to a noise power $\sim 30$ MW without input signal. Fig. 9 shows that the rear edge of the input pulse is constantly fed by electrons without initial modulation (excepting the effect of noise). In this situation, the saturation mechanism typical for amplification of a monochromatic signal does not work. Therefore, at this rear edge the intensive growth of radiation amplitude takes place. For the simulation parameters, the power amplification coefficient is $\sim 8$. This process is accompanied by significant shortening of the input pulse duration by four to five times. It is important that the amplitude of the input pulse is close to the saturation value for the case of amplification of a stationary monochromatic signal. Nevertheless, significant pulse amplification is observed.

The influence of such effects as dispersive expansion, spread of particle velocities, space charge etc., are investigated

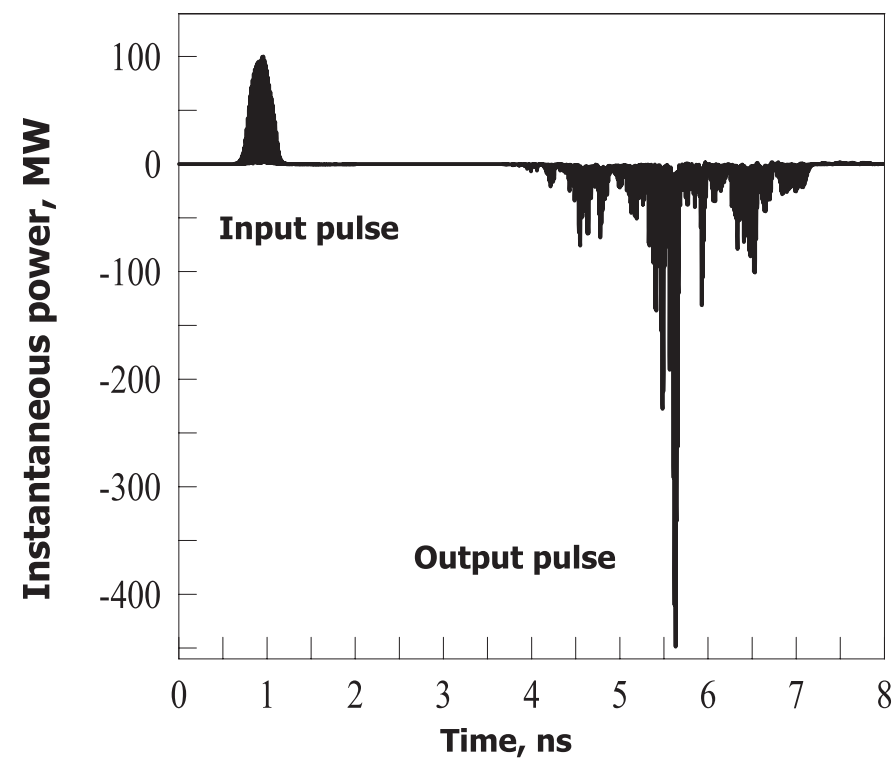

Fig. 10. KARAT simulation of amplification of SR pulse by electron beam in dielectric loaded waveguide in conditions close to experimental ones.

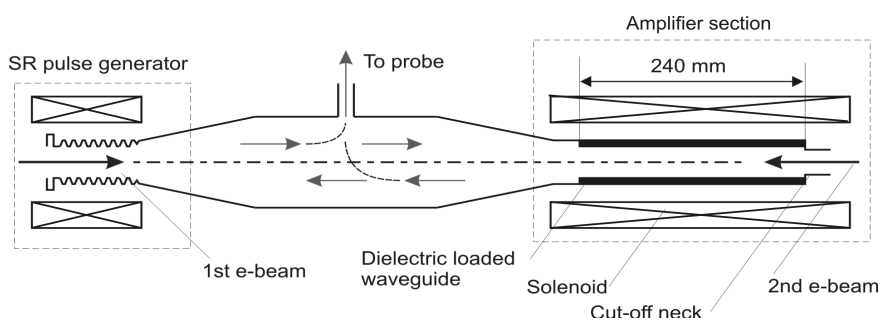

Fig. 11. Experimental setup for observation of amplification and compression of SR pulses.

using the PIC code KARAT. The simulation result of the amplification of a $50 \mathrm{MW}, 300-\mathrm{ps}$ input pulse is shown in Fig. 10. The output power achieves $210 \mathrm{MW}$ (amplification coefficient $\sim 4.2$ ). Simultaneously, significant shortening of the input pulse down to 80 ps occurs. In the absence of an input signal the multispikes regime (SASE) is realized with a power level of 30-40 MW (noise pedestal in Fig. 10 with duration $\sim 2 \mathrm{~ns})$. According to the simulations, when the interaction space length is increased up to $30 \mathrm{~cm}$ the output peak power reaches $\sim 350 \mathrm{MW}$, exceeding the power of the electron beam [33].

\section{B. Experimental Observation of Amplification and Nonlinear Compression of SR Pulse at Ka-Band}

For observation of amplification and compression of SR pulses the experimental setup (Fig. 11) based on two RADAN accelerators, synchronized with accuracy $100-300$ ps [16] is arranged. The first accelerator is used to drive the $37-\mathrm{GHz}$ generator of SR pulses with duration $\sim 300$ ps based on a relativistic BWO. This SR pulse is transmitted through the diagnostic oversized waveguide to the amplification section formed by a dielectric loaded waveguide. After reflection from a cutoff region, the SR pulse is amplified by an electron beam with current $1.2 \mathrm{kA}$ and duration up to $2.5 \mathrm{~ns}$ from a second RADAN accelerator. 
Recording of the input and output pulses are performed in the diagnostic section by a calibrated hot-carrier germanium detector. The signals are recorded by a transient digitizing oscilloscope Tektronix TDS-6154C (15 GHz; $40 \mathrm{Gs} / \mathrm{s})$. At the preliminary stage of the experiments, the attenuation coefficients for microwave signals with durations from 100 to $300 \mathrm{ps}$ are estimated. Based on these considerations for a SR pulse peak power $\sim 130 \mathrm{MW}$ the power at the input of the amplification section should not exceed $50 \mathrm{MW}$ [Fig. 12(a)]. The main reasons for the power reduction are parasitic reflection in the transmission line, as well as RF breakdown.

In the absence of an input signal from the SR pulse generator the regime of self-amplification of spontaneous emission is realized with duration $\sim 2-2.5 \mathrm{~ns}$ and power level 30 $40 \mathrm{MW}$ [Fig. 12(b)]. When the amplification section is driven by an input SR pulse a short single amplified output pulse is observed, as shown in Fig. 12(c). The peak power of the output pulse is $\sim 190 \mathrm{MW}$ (power amplification gain $\sim 3.8$ ). Amplification is accompanied by significant shortening of the electromagnetic pulse duration down to $100 \mathrm{ps}$.

Thus, the performed experiments demonstrated the amplification of microwave SR pulses propagating along quasistationary electron beams, which is accompanied by a strong shortening (nonlinear compression) of the pulses. However, the peak power of generators of SR pulses exceeds the gigawatt level [21], [22]. To effectively amplify such pulses it is necessary to drive the amplification section by a high-current electron beam with a power much higher than the beam power in the generator of the SR pulses. Nevertheless, even at the present stage of experiments, the duration of the observed subgigawatt pulses of $\sim 100 \mathrm{ps}$ is a record value for the Ka-band and corresponds to 5-6 periods of high-frequency oscillations.

\section{Sit and Electromagnetic Pulse Compression in Plasma or Electron Beam Under Cyclotron RESONANCE CONDITION}

The effect of SIT for short (in the scale of relaxation times) light pulses is well-known in optics and is studied in detail theoretically and experimentally [3]-[5]. Because of nonlinear effects, a dissipative medium that normally absorbs light becomes transparent to a bright, short-duration light pulse ( $2 \pi$-pulse). Therefore, the light pulse propagates without any change in shape as a SIT-soliton. In addition, under certain conditions nonlinear compression of the incident light pulse occurs when its duration reduces while the amplitude significantly increases [5]. Similar effects can, apparently, be observed in classical electronics when a short electromagnetic pulse propagates through an initially rectilinear electron beam under the cyclotron resonance condition. In the condition of the normal Doppler effect $\beta_{p h}^{-1} \beta_{\| 0}<1$ (where $v_{p h}=\beta_{p h} c$ is the phase velocity of the radiation) such beams can be considered as an analogy of a noninverted (passive) two-level medium.

\section{A. Model and Basic Equations}

In principle, SIT effects should be observed for arbitrary angles between the pulse group velocity and the direction of a

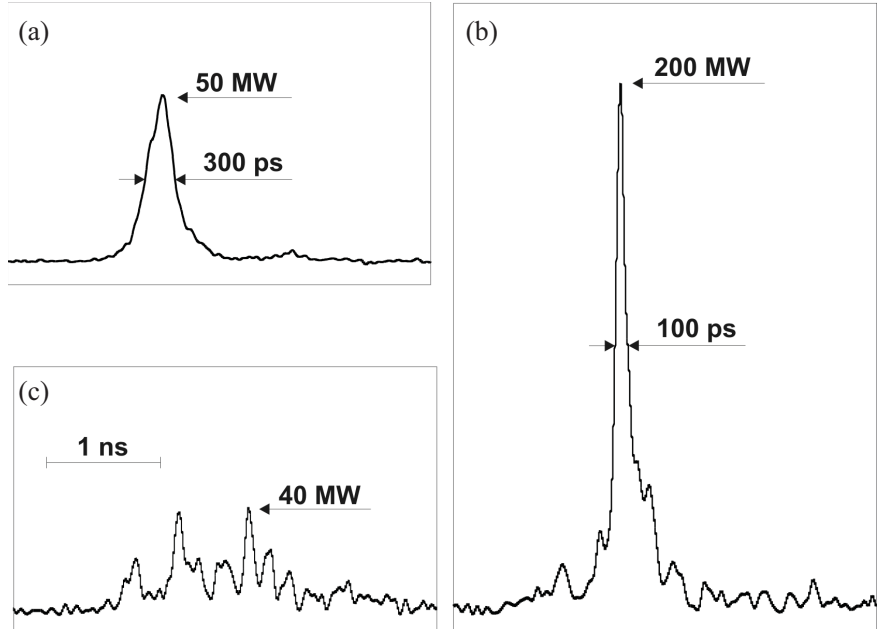

Fig. 12. Experimental results on observation of Cherenkov amplification of SR pulse in Ka-band. (a) Typical input pulse from generation of SR pulses. (b) Multispike regime in absence of input pulse. (c) Amplified output pulse.

homogeneous magnetic field: $\vec{H}=\vec{z}_{0} H_{0}$. Let us consider the collinear propagation of an electromagnetic pulse with respect to the electrons' translational velocity in a waveguide. Under the cyclotron resonance condition

$$
\omega \mp h v_{\| 0} \approx \omega_{H}
$$

where $\omega_{H}=e H_{0} / m c \gamma$ is the gyrofrequency, the interaction can be described by the following:

$$
\begin{aligned}
& \frac{\partial \hat{a}}{\partial \hat{z}} \pm \frac{1}{\beta_{\mathrm{gr}}} \frac{\partial \hat{a}}{\partial \hat{t}}=\mp G \frac{\hat{p}_{+}}{1 \pm b\left|\hat{p}_{\perp}\right|^{2}} \\
& \frac{\partial \hat{p}_{+}}{\partial \hat{z}}+\frac{1}{\beta_{\| 0}} \frac{\partial \hat{p}_{+}}{\partial \hat{t}}+i \hat{p}_{+} \frac{\Delta+\mu\left|\hat{p}_{+}\right|^{2}}{1 \pm b\left|\hat{p}_{+}\right|^{2}}=\frac{\hat{a}}{1 \pm b\left|\hat{p}_{+}\right|^{2}} .
\end{aligned}
$$

Here, we use the following dimensionless variables and parameters

$$
\hat{t}=\omega t, \quad \hat{z}=\frac{\omega z}{c}, \hat{a}=\frac{i A E_{S}\left(R_{0}\right)\left(1 \mp \beta_{p h}^{-1} \beta_{\| 0}\right)}{m c \omega \beta_{\| 0}^{2}}
$$

where $p_{+}=\left(p_{x}+i p_{y}\right) \exp (i \omega t \mp i h z) / m c \gamma_{0} \beta_{\| 0}$ is the transverse momentum of electrons, $\mu=\beta_{\| 0}\left(1-\beta_{p h}^{-2}\right) / 2(1 \mp$ $\left.\beta_{p h}^{-1} \beta_{\| 0}\right)$ is the parameter of nonisochronicity, $b=\beta_{p h}^{-1} \beta_{\| 0} /$ $2\left(1 \mp \beta_{p h}^{-1} \beta_{\| 0}\right)$ is the variation of the longitudinal momentum $\hat{p}_{\|}=p_{\|} / m c \gamma_{0} \beta_{\| 0}=1 \mp b\left|\hat{p}_{+}\right|^{2}, \Delta=\left(1 \mp \beta_{p h}^{-1} \beta_{\| 0}-\omega_{H 0} /\right.$ w) $\beta_{0}$ is the initial mismatch of the cyclotron resonance, and

$$
G=4 \frac{e I_{b}}{m c^{3}} \frac{\left(1 \mp \beta_{p h}^{-1} \beta_{\| 0}\right)}{\gamma_{0} \beta_{p h}^{-1} \beta_{\perp 0}^{4}} \frac{J_{m-1}^{2}\left(\kappa r_{b}\right)}{\left(v_{n}^{2}-m^{2}\right) J_{m}^{2}\left(v_{n}\right)}
$$

is a form factor written under the assumption that the electron bunch interacts with a $T E_{m n}$ mode of the waveguide with radius $R, \kappa=v_{n} / R, v_{n}$ is the $n$th root of the equation $J_{m}^{\prime}(v)=0$, and $J_{m}$ is a Bessel function. The upper sign in the above equations corresponds to the interaction of electrons with the co-propagating incident pulse and the lower sign corresponds to the case of counter-propagation. 
It is important that a fundamental factor for the development of the SIT effects is a nonzero nonisochronicity parameter $\mu$. As in the physics of gyrotrons [38], nonisochronicity is caused by a relativistic dependence of the gyrofrequency on the particle energy. However, collinear propagation of the wave relative to the magnetic field it can be partially compensated by recoil effects. Under the autoresonance condition (when the phase velocity is equal to the speed of light) full compensation takes place $\mu=0$. In this case, electron oscillators convert to linear ones. Correspondingly, SIT effects develop only out of autoresonance when $\mu \neq 0$.

\section{B. SIT and Compression of Electromagnetic Pulses Under Interaction With a Co-Propagating Electron Beam}

Let us consider the situation when an incident electromagnetic pulse propagates in the forward direction with respect to the electron longitudinal velocity. Under the assumption of small electron current $G \ll 1$ and nonzero parameter of nonisochronicity (13) can be reduced to the following form:

$$
\begin{aligned}
\frac{\partial a}{\partial Z}+\frac{\partial a}{\partial \tau} & =-p \\
\frac{\partial p}{\partial Z}+i p\left(\delta+|p|^{2}\right) & =a .
\end{aligned}
$$

where $\tau=\sqrt{G}\left(\hat{t}-\hat{z} / \beta_{\| 0}\right)\left(\beta_{\mathrm{gr}}^{-1}-\beta_{\| 0}^{-1}\right)^{-1}, Z=\sqrt{G} \hat{z}$, $p=\hat{p}_{+} \mu^{1 / 2} G^{-1 / 4}, a=\hat{a} \mu^{1 / 2} G^{-3 / 4}$, and $\delta=\Delta G^{-1 / 2}$. The equations for electron motion are presented as equations of nonisochronous oscillators which are widely used in the theory of CRMs [38]. The main difference between the model considered here and the traditional models of gyroresonance devices lies in the assumption that the electrons have no initial rotational velocity: $p(Z=0)=0$. Therefore, a resonant medium is described by (14b), i.e., no averaging over the cyclotron rotation phases is required.

The results of the solutions of (14) are shown in Fig. 13 for different initial amplitudes $a_{0}$ and durations $T$ of the incident electromagnetic pulse. These results confirm the analogy with the optical SIT effects. When the amplitude and duration of the incident electromagnetic pulse are small, the cyclotron absorption is shown in [Fig. 13(a)]. This process is accompanied by a quasi-periodical energy exchange between the electromagnetic pulse and the electrons that is similar to Rabi oscillations of population inversion in optics [7]. Following the above analogy, as the initial amplitude and duration of the microwave pulse increase, the SIT effect occurs [35], [36] when the incident pulse propagates through electron beam practically without damping [Fig. 13(b)]. This effect has a simple explanation; viz. the leading front of an electromagnetic pulse excites transverse oscillations of electrons which then are suppressed by the trailing front. With further increasing of the amplitude and duration of the incident pulse the nonlinear pulse compression is observed. In Fig. 13(c), the peak power grows $>2.5-2.8$ times with simultaneous pulse shortening. In this process, the energy absorption is rather low and the energy of the compressed pulse amounts to $80 \%$ of the incident pulse energy. Similar to the propagation of light pulses in the resonance two-level medium, for sufficiently large amplitude

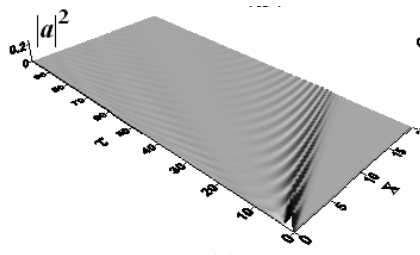

(a)

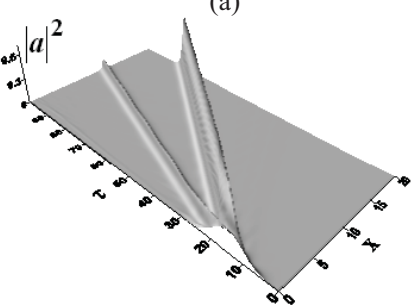

(c)
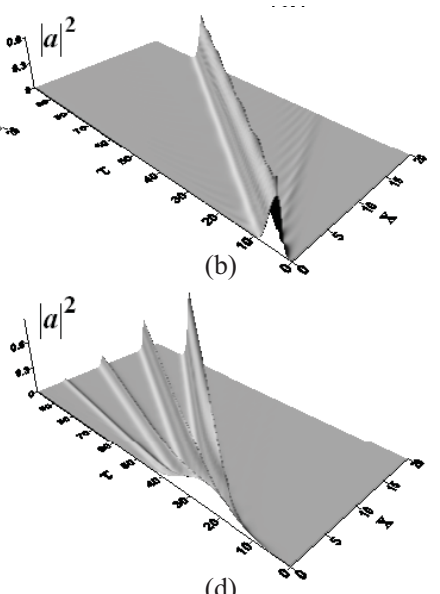

(d)

Fig. 13. Effects arising in cyclotron resonance condition under interaction of short electromagnetic pulse with co-propagating electron beam with varying peak amplitude and duration of incident pulse. (a) Cyclotron absorption of pulse $\left(a_{0}=0.5, T=3\right)$. (b) Effect of SIT $\left(a_{0}=0.7, T=10\right)$. (c) Nonlinear compression of input pulse $\left(a_{0}=0.5, T=30\right)$. (d) Decay of input pulse on several solitons $\left(a_{0}=0.7, T=40\right)$.

and duration the incident electromagnetic pulse decomposes to one, or several, solitons, e.g., localized wave packets with amplitude and duration defined by the velocity of their propagation.

Soliton-like solutions of (14) can be found analytically and have the following form [35]:

$$
|a(Z, \tau)|=\frac{2}{s^{3 / 4}}\left[\operatorname{sech} \frac{2}{\sqrt{s}}(Z-U \tau)\right]^{1 / 2}
$$

where $s=U-1$ and $U$ are the normalized soliton velocity. According to (15), the soliton amplitude increases with simultaneous shortening of its duration with decreasing of the parameter $s$, e.g., with decreasing the difference between the soliton velocity and the velocity of light. These conclusions are in a good agreement with the results of numerical simulations of time-domain (14) [see Fig. 13(d)]. Optical SIT-solitons possess a similar dependence of amplitude and duration on propagation velocity.

\section{Stopping of an Electromagnetic Pulse Interacting With a Counter-Propagating Electron Beam}

The SIT and pulse compression effects can also take place when the radiation group velocity is directed backward to the electron translational velocity. A distinctive feature in counter interaction is the possibility of the stopping of an electromagnetic pulse and the formation of a stationary soliton. The system of equations describing the electron-wave interaction in wave counter propagation relative to the electron beam can be presented in the form

$$
\begin{aligned}
\frac{\partial a}{\partial Z}-\frac{\partial a}{\partial \tau} & =p \\
\frac{\partial p}{\partial Z}+i p\left(\delta+|p|^{2}\right) & =a
\end{aligned}
$$




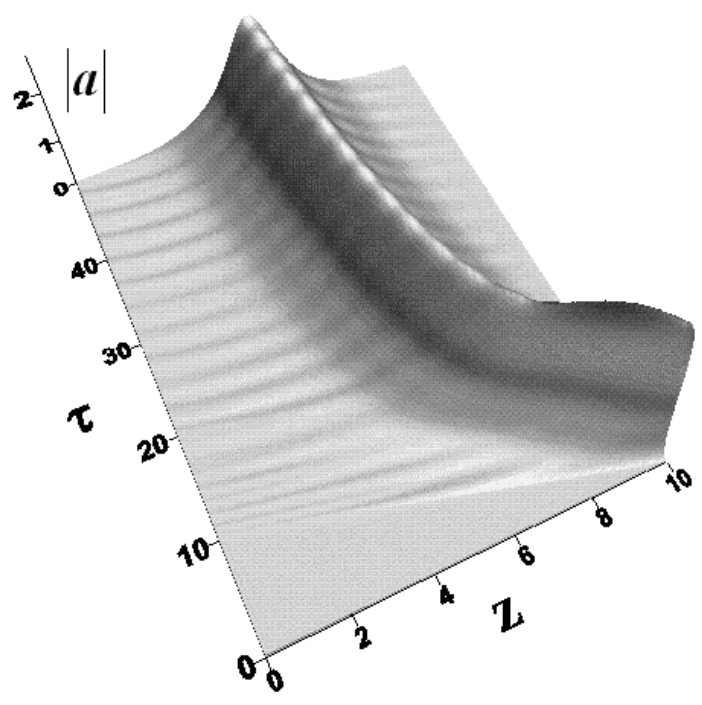

Fig. 14. Stopping of incident electromagnetic pulses in cyclotron resonance condition under interaction with counter-propagating electron beam.

where $\tau=\sqrt{G}\left(\hat{t}-\hat{z} / \beta_{\| 0}\right)\left(\beta_{\text {gr }}^{-1}+\beta_{\| 0}^{-1}\right)^{-1}$ is used as the time variable. Similar to (14), we assume that the electrons at the entrance into the interaction space $Z=0$ have no transverse momentum. The (16) also have a soliton-like solution that coincides with (15) if we set $s=U+1$. Therefore, a soliton interacting with a counter propagating electron beam exists under the condition $U>-1$. The soliton velocity is directed in the opposite direction to that of the electron beam in the range $-1<U<0$ and in the forward direction in the range $U>0$. Obviously, the soliton velocity can also be zero, $U=0$, which corresponds to the stopping of an electromagnetic pulse [36].

These conclusions are confirmed by numerical simulations of (16). Fig. 14 shows deceleration and stopping of an electromagnetic pulse when it propagates from the collector end of the system. We see that in electromagnetic pulse propagates in the opposite direction to the electron beam with an unperturbed group velocity, but then it decelerates to the point of full stopping as it transforms into a soliton. In contrast to the stopping of light realized through the photoinduced transparency effects [39]-[41], the considered case the stopping occurs because of movement of the nonlinear resonance medium in the frame of a more simple single photon process (e.g., in the absence of a pump wave).

In the case of interaction with a counter propagating electron beam, the regimes described above can also be realized including the cyclotron absorption, SIT, the formation of multisoliton solutions, and the compression of an input signal. The nonlinear compression effect can be used for further increasing the peak power and shortening the duration of SR pulses. Let us estimate the possibility of compressing an 8-mm SR pulse with a power of $1.2 \mathrm{GW}$ and a duration of $350 \mathrm{ps}$ [22] propagating in a waveguide with radius of $0.45 \mathrm{~cm}$ in the opposite direction to an electron beam with a particle energy of $300 \mathrm{keV}$ and a current of $450 \mathrm{~A}$. We will assume that the transverse structure of the signal corresponds to the $\mathrm{TE}_{11}$ mode of a circular waveguide. The wave phase velocity $v_{\mathrm{ph}} \approx 1.2 c$. The electron

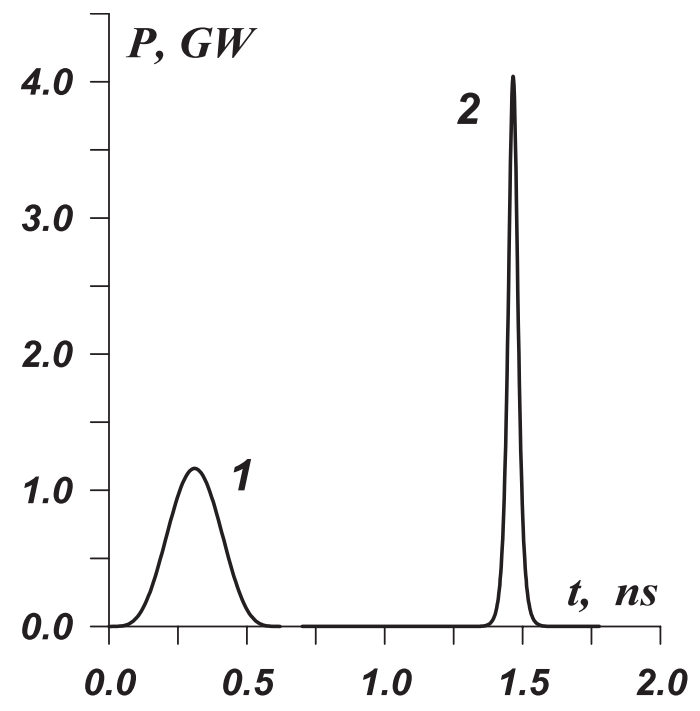

Fig. 15. Simulation of nonlinear compression of SR pulse in cyclotron resonance condition. (1) Incident. (2) Compressed microwave pulses.

beam is guided by a uniform magnetic field with strength of 3.5 $\mathrm{T}$, which provides the condition for cyclotron resonance with the radiation of the above band. At the chosen physical parameters, the normalized parameters are $G=0.008, a_{0}=5$, and $T=6$. The input and output pulses at the optimal cyclotron resonance detuning $\delta=2$ are shown in Fig. 15. The peak power of the output pulse is $\sim 3.3 \mathrm{GW}$ at a duration of $\sim 80 \mathrm{ps}$. The normalized length of the compression region $L=10$ corresponds to a physical length of $\sim 10 \mathrm{~cm}$. Thus, the investigated process can be fairly efficient in practice for the generation of electromagnetic pulses with an ultrahigh peak power and an ultrashort duration. The proposed method may be considered as an alternative to the well-known methods of passive and active compression [42], [43] in a certain domain of parameters.

The deceleration and stopping of electromagnetic pulses can be of practical interest, for example, for their subsequent addition or the creation of delay lines. For full stopping of an SR pulse with the above power and duration to be observed, the beam current must be increased to $1 \mathrm{kA}$. Obviously, the experimental setup based on two synchronized RADAN accelerators, for which the nonstationary amplification of SR pulses is investigated [34], can be used to observe the effects being discussed after modification. For this purpose, the amplifier section in the form of a waveguide with a dielectric insert should be replaced by a section in the form of a regular waveguide, whereas the strength of the guiding magnetic field should correspond to the cyclotron resonance of particles with a co-propagating or counter-propagating wave.

\section{Generation of Terahertz SR Pulses by Multipicosecond Electron Bunches Moving Over a CORRugated Surface}

This section is describes the analysis of possibilities for the development of SR sources in short wavebands including terahertz one. We study the Cherenkov mechanisms of SR on the assumption that the electron bunches are formed by photoinjectors [44], [45]. Advancement of Cherenkov SR 
sources into short-wave ranges needs fabrication of periodic microstructures based on modern technologies. Increasing the electron energy up to several $\mathrm{MeV}$, that is typical for electron bunches formed by photoinjectors, allows the achievement of the proper coupling with an evanescent wave. In contrast with the previous sections strongly oversized waveguide structures should be used in the terahertz frequency range leading to the necessity to develop a quasi-optical approach for the description of Cherenkov radiation.

Let us consider the Cherenkov SR of an extended electron bunch of finite length $l_{z}^{e}$ and transverse dimensions $l_{x, y}^{e}$ that moves rectilinearly over a plane with a periodic sinusoidal corrugation: $b(z)=b_{1} \cos (\bar{h} z)$, where $b_{1}$ is the corrugation amplitude [Fig. 16(a)]. As shown in [46], if the corrugation depth is relatively small, the formation of an evanescent slow wave over the corrugated surface can be represented as a superposition of two counter-propagating quasi-optical wavebeams that defines by the magnetic field

$H_{x}=\operatorname{Re}\left[A_{+}(z, x, y, t) e^{i(\omega t-k z)}+A_{-}(z, x, y, t) e^{i(\omega t+k z)}\right]$

where $k=\omega / c$. Accordingly, the longitudinal component of the electric field that acts upon the electrons can be expressed as follows:

$$
E_{z}=-\operatorname{Re} \frac{i}{k}\left[\frac{\partial A_{+}}{\partial y} e^{i(\omega t-k z)}+\frac{\partial A_{-}}{\partial y} e^{i(\omega t+k z)}\right] .
$$

On a corrugated surface under the Bragg resonance conditions $\omega=\bar{h} c / 2$, the counter-propagating waves (17) exhibit coupling and mutual scattering because of excitation of virtual surface magnetic currents [47]. Therefore, an evanescent slow wave is formed with dispersion characteristic shown in Fig. 16(b). For relativistic energies the intersection of the dispersion curve of a normal slow wave with the eigenwave of a rectilinear electron beam: $\omega=h V_{\| 0}$ corresponds to a regime in which the direction of normal wave group velocity coincides with the electron longitudinal velocity.

In the frame of a 3-D quasi-optical model the process of SR can be described by the following system:

$$
\begin{aligned}
& \frac{\partial \hat{A}_{+}}{\partial Z}+\frac{\partial \hat{A}_{+}}{\partial \tau}+i \frac{\partial^{2} \hat{A}_{+}}{\partial X^{2}}+i \frac{\partial^{2} \hat{A}_{+}}{\partial Y^{2}}=i \hat{\alpha} \hat{A}_{-} \delta(Y) \\
& \quad-\frac{\chi\left(\tau-Z / \beta_{\| 0}\right)}{B_{e}} \frac{\partial}{\partial Y}(J F(X, Y)) \\
& -\frac{\partial \hat{A}_{-}}{\partial Z}+\frac{\partial \hat{A}_{-}}{\partial \tau}+i \frac{\partial^{2} \hat{A}_{-}}{\partial X^{2}}+i \frac{\partial^{2} \hat{A}_{-}}{\partial Y^{2}}=i \hat{\alpha} \hat{A}_{+} \delta(Y)
\end{aligned}
$$

where $\chi\left(\tau-Z / \beta_{\| 0}\right)$ is the function that describes the unperturbed bunch longitudinal profile, $B_{e}=\int_{-\infty}^{+\infty} \int_{0}^{\infty}$ $F(X, Y) d X d Y$ is the effective thickness of the electron bunch, and $F(X, Y)$ is the transverse distribution of the electron density. We assume a uniform transverse distribution: $F(X, Y)=1$ for $Y \in\left[B_{g}, B_{g}+B_{e}\right]$ and $X \in$ $\left[-L_{x}^{e} / 2, L_{x}^{e} / 2\right]$, where $B_{g}$ is the gap between the bunch and the corrugation and $L_{x}^{e}$ is the dimensionless width of the electron bunch. The RF electron current $J(Z, X, Y, \tau)=$ $(1 / \pi) \int_{0}^{2 \pi} \exp (-i \theta) d \theta_{0}$ can be found from the electron

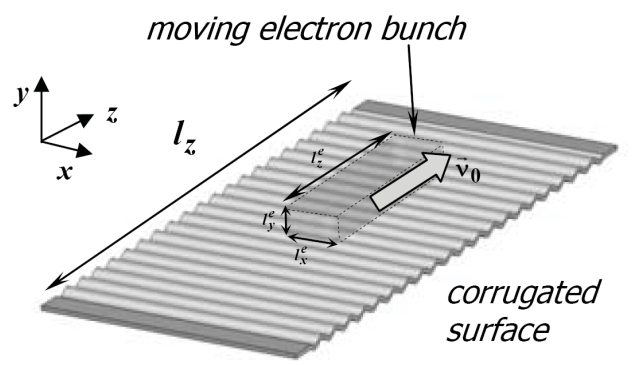

(a)

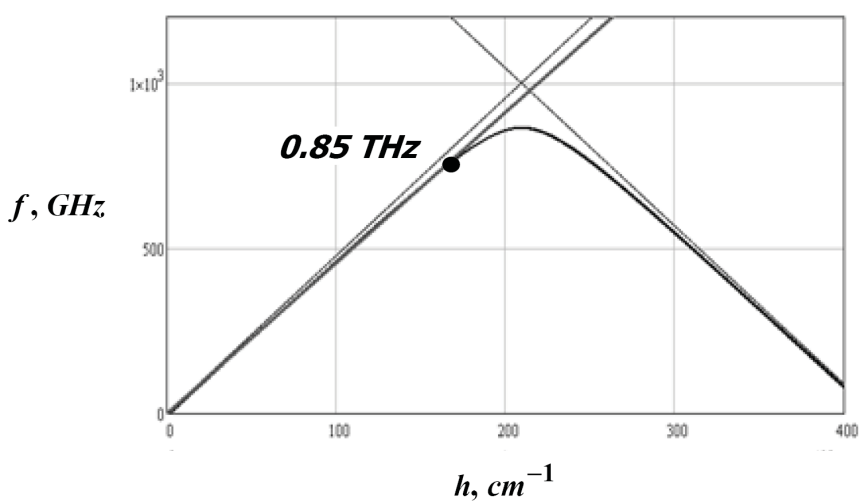

(b)

Fig. 16. (a) Principal scheme of generation of SR pulses by extended electron bunch moving over periodically corrugated surface. (b) Dispersion diagram of normal surface wave and resonance point in case of relativistic rectilinear electron beam.

motion equations

$$
\begin{aligned}
& \left(\frac{\partial}{\partial Z}+\frac{1}{\beta_{\| 0}} \frac{\partial}{\partial \tau}\right)^{2} \theta=\operatorname{Re}\left(\frac{\partial \hat{A}_{+}}{\partial Y} e^{i \theta}\right) \\
& \left.\theta\right|_{Z=0}=\theta_{0} \in[0,2 \pi),\left.\quad\left(\frac{\partial}{\partial Z}+\frac{1}{\beta_{\| 0}} \frac{\partial}{\partial \tau}\right) \theta\right|_{Z=0}=\Delta
\end{aligned}
$$

where $\theta=\omega_{0}(t-z / c)$ is the phase of electrons relative to the co-propagating partial wave $A_{+}$. The system of (19), (20) is written using the following normalization: $Z=G k z, X=\sqrt{2 G} k x, Y=\sqrt{2 G} k y, \tau=G \omega t$, $\hat{A}_{ \pm}=e \mu A_{ \pm} /\left(m c \omega \gamma_{0} G^{3 / 2}\right), \hat{\alpha}=\bar{h} b_{1} / 2 \sqrt{2 G}, G=$ $\left(2 \sqrt{2}\left(\left(e I_{b} / m c^{3}\right)\left(\mu \lambda /\left(\gamma_{0} l_{x}^{e}\right)\right)\right)\right)^{2 / 3}$ is the gain parameter, and $\mu=\gamma_{0}^{-2}$ is the parameter of inertial electron bunching, $\hat{\Delta}=2 \Delta / \bar{h} G$. The boundary conditions to (19) correspond to the absence of electromagnetic energy fluxes from outside: $\left.\hat{A}_{+}\right|_{Z=0}=0,\left.\hat{A}_{-}\right|_{Z=L}=0$, where $L=G k l$. At the boundaries of the corrugation along the $x$ coordinate nonreflected radiation conditions should be applied.

Simulations of the Cherenkov SR are performed in the terahertz range for the electron bunch with the length of $l_{z}^{e}=1.2 \mathrm{~cm}(40 \mathrm{ps})$, the transverse dimensions of $l_{x}^{e}=l_{y}^{e}=$ $0.3 \mathrm{~mm}$, particle energy of $1.5 \mathrm{MeV}$ and total charge of $2.2 \mathrm{nC}$. These parameters can be obtained for the electron bunches generated by photoinjectors [44], [45]. The bunch is propagating over a plane with a corrugated region of length $10 \mathrm{~cm}$ with corrugation period of $0.15 \mathrm{~mm}$, and amplitude of $25 \mu \mathrm{m}$. These physical parameters correspond to the normal- 


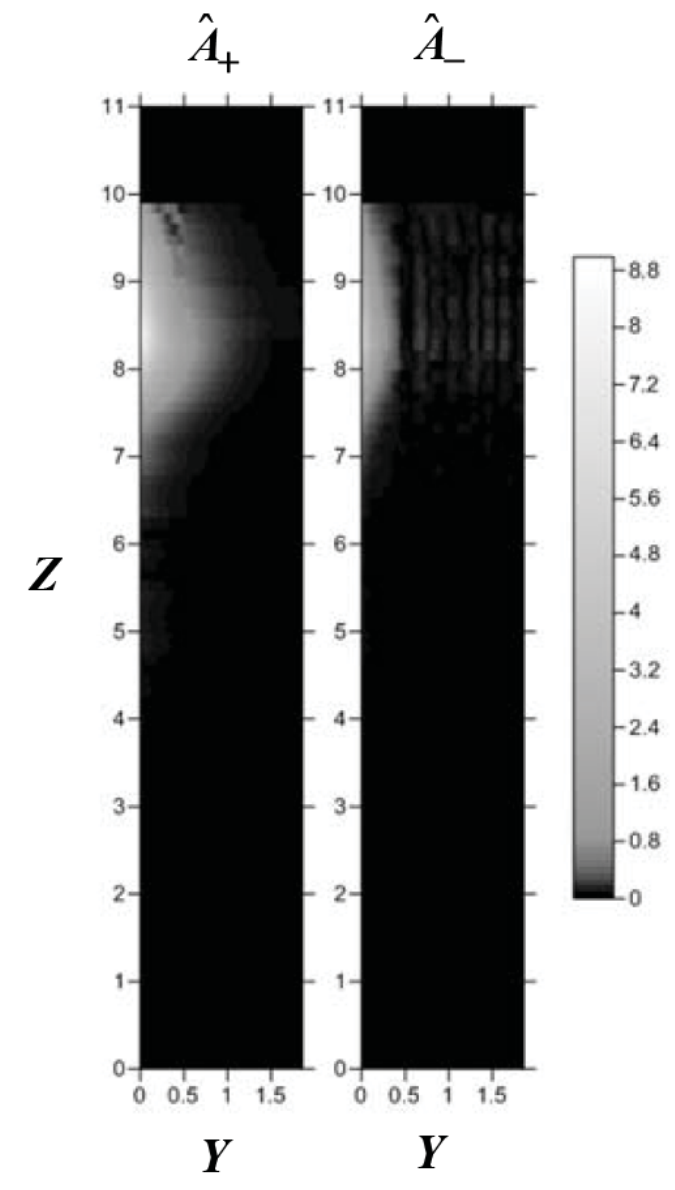

Fig. 17. Spatial structures of partial waves in cross section $X=0$.

ized quantities $G=2.4 \cdot 10^{-3}, \hat{\alpha}=7.4, \Delta=15, B_{e}=0.45$, $L=8$, and $L_{x}^{e}=0.67$. It is assumed that, at the entrance of the interaction space (initial moment of time), electrons are uniformly (to within small fluctuations) distributed over the bunch with a normalized duration: $T_{e}=G \omega_{0} l_{e} / v_{\| 0}=0.64$ $\left(\chi(\tau, 0)=1, \tau \in\left[0, T_{e}\right]\right)$.

The results of solutions to the differential equations showed that a short SR pulse emits in the positive direction of the axis $z$, i.e., in the direction of the electron bunch propagation. Fig. 17 shows exponential decay of the amplitudes of both partial waves in the vertical $y$ direction that obviously corresponds to formation of an evanescent slow wave. In Fig. 18, the instant electric field $E_{y}(X, Z)$ space profiles in the cross section $Y=0.6$ are shown in consecutive time intervals. From the dispersion diagram, the electron velocity in the resonance point exceeds the normal wave group velocity. Therefore, Fig. 18 shows the formation of wake-fields behind the electron bunch. The peak power of the SR pulse amounted to $3.5 \mathrm{MW}$ at a pulse duration of $\sim 100 \mathrm{ps}$.

Results obtained in the framework of a quasi-optical model are confirmed by the direct PIC simulations based on the code CST Studio that are shown in Fig. 19(a) and (b) for the physical parameters of the SWS system and the electron bunch indicated above. The SR pulse peak power is $\sim 3 \mathrm{MW}$, pulse duration is $60 \mathrm{ps}$, and the spectrum central frequency is $\sim 0.8 \mathrm{THz}$. Fig. 19(c) also shows formation of wake-field behind the electron bunch.
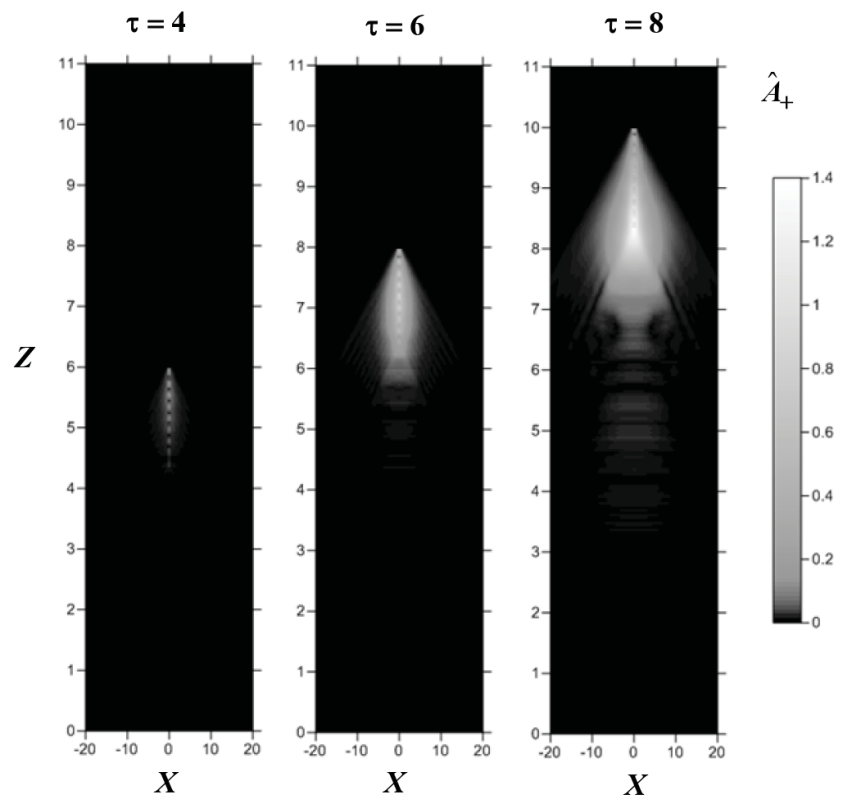

Fig. 18. Formation of wake wave after electron bunch. Transverse $(X, Z)$ profiles of electric field on surface $Y=0.6$ are shown in consecutive points in time.

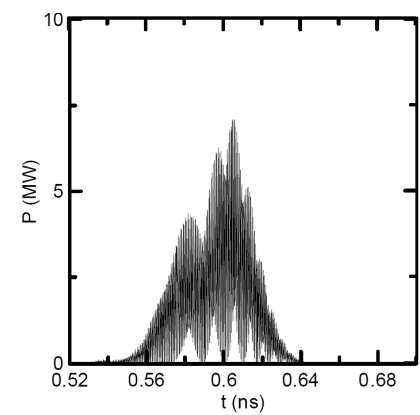

(a)

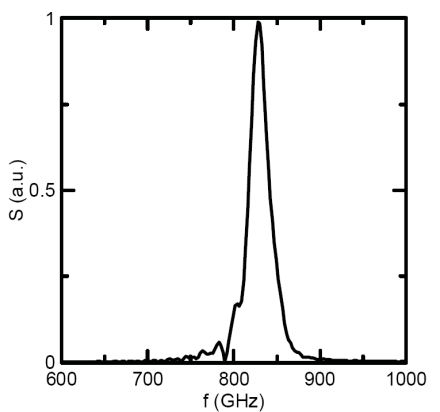

(b)
$\left|E_{y}\right|$

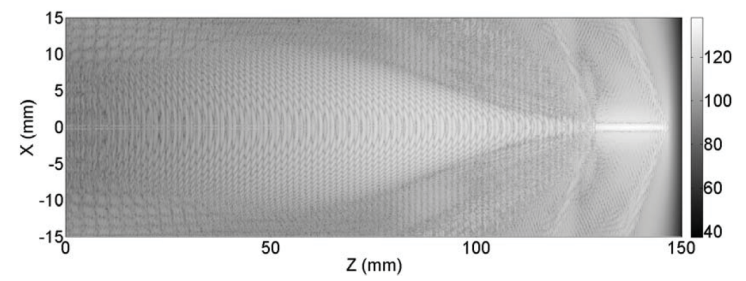

(c)

Fig. 19. Results of 3-D direct PIC simulations of Cherenkov SR in terahertz range for electron bunch formed by photoinjector gun. (a) SR pulse. (b) Radiation spectrum. (c) Formation of wake-field.

Thus, the results of our analysis demonstrate the possibility of generation of single high-power electromagnetic pulses in the terahertz frequency range using Cherenkov SR of electron bunches that propagate in free space over a corrugated surface. We believe that it is expedient to study the possibility of moving to still shorter wavelengths, which can be achieved by decreasing the period of the gratings with simultaneous increasing the density and particle's energy. We analyzed the generation of terahertz SR pulses by electron bunches moving in an undulator field. For closely similar parameters of the electron bunches and the generated SR pulses, evident 
advantages of the Cherenkov mechanism are a smaller size of the interaction space and the possibility of using electron bunches with lower particle energies (for the same radiation frequency).

In many papers, the radiation of an electron bunch moving above a corrugated surface is described as the Smith-Purcell effect [48]-[50]. Although spontaneous emission was studied in these papers, they did not include the electrons' microbunching. This type of emission is effective when the bunch size is small on the scale of a wavelength. It is typical for such a type of emission that the electrons simultaneously radiate at many frequencies that depend on the observation angle. Our model describes stimulated emission and (19), (20) obviously include the electrons' microbunching. In contrast to spontaneous emission, stimulated emission is usually characterized by a narrow frequency band. The radiation frequency is close to the Bragg frequency, i.e., the radiation wavelength is approximately half of the corrugation period. For the Smith-Purcell effect the frequencies radiated at a small angle to the direction of the electron longitudinal velocity can significantly exceed the Bragg frequency. However, the conditions for providing stimulated emission at such high frequencies still need more analysis.

\section{CONCLUSION}

The theoretical understanding and experimental demonstration of SR were reviewed and showed to be a very effective method of producing very high-power short pulses of electromagnetic radiation from electron bunches. Types of SR associated with different mechanisms (cyclotron, Cherenkov, and bremsstrahlung) of stimulated emission were analyzed theoretically, numerically modeled using PIC codes, and observed experimentally. Cherenkov SR in the configuration of an electron bunch interacting with a backward wave produced some of the highest peak powers. The earliest SR experiments of this type used uniform SWSs. SR experiments used nonuniform SWSs and produced SR pulses with peak power exceeding the electron beam power. Recent experiments achieved phase synchronization of two SR pulse generators opening the way for synchronizing multiple sources and lead to the prospect of steerable SR beams from phased arrays of SR generators.

The simulation and experimental observation of amplification of SR pulses during propagation along quasi-stationary electron beams were reviewed. In addition, an alternative method was presented for further increasing the SR pulse peak power associated with nonlinear compression in the process of induced self-transparency. Finally, the development of SR sources for higher frequency bands was considered as an effective method of production of ultrashort electromagnetic pulses.

In summary, progress in SR research enabled a new type of generator to be created capable of generating unique short (under 200-300 ps) electromagnetic pulses at super high peak powers exceeding $1 \mathrm{GW}$ in the millimeter and $3 \mathrm{GW}$ in the centimeter waveband. In addition, it was showed that Cherenkov SR of short electron bunches that propagated in free space over a corrugated surface should be capable of generating several $\mathrm{MW}$ at $\mathrm{THz}$ frequencies.

\section{REFERENCES}

[1] R. H. Dicke, "Coherence in spontaneous radiation processes," Phys. Rev., vol. 93, no. 1, pp. 99-110, Jan. 1954.

[2] J. C. MacGillivray and M. S. Feld, "Theory of superradiance in an extended, optically thick medium," Phys. Rev. A, vol. 14, no. 3, pp. 1169-1189, Sep. 1976.

[3] N. Scribanowitz, I. P. Hermann, J. C. MacGillivray, and M. S. Feld, "Observation of dicke superradiance in optically pumped HF gas," Phys. Rev. Lett., vol. 30, no. 8, pp. 309-312, Feb. 1973.

[4] S. L. McCall and E. L. Hahn, "Self-induced transparency by pulsed coherent light," Phys. Rev. Lett., vol. 18, no. 21, pp. 908-911, May 1967.

[5] S. L. McCall and E. L. Hahn, "Self-induced transparency," Phys. Rev., vol. 183, no. 2, pp. 457-485, Jul. 1969.

[6] H. M. Gibbs and R. E. Slusher, "Optical pulse compression by focusing in a resonant absorber," Appl. Phys. Lett., vol. 18, no. 11, pp. 505-507, Jun. 1971.

[7] L. Allen and J. H. Eberly, Optical Resonance and Two-Level Atoms, New York, USA: Wiley, 1975.

[8] J. P. Wittke and P. J. Warter, "Pulse propagation in a laser amplifier," J. Appl. Phys., vol. 35, no. 6, pp. 1668-1672, Jun. 1964.

[9] R. H. Bonifacio, C. Maroli, and N. Piovella, "Slippage and superradiance in the high gain FEL-linear theory," Opt. Commun., vol. 68, no. 5, pp. 369-374, Nov. 1988.

[10] R. H. Bonifacio, N. Piovella, and B. W. J. McNeil, "Superradiant evolution of radiation pulses in a free-electron laser," Phys. Rev. A, vol. 44, no. 6, pp. R3441-R3444, Sep. 1991.

[11] N. S. Ginzburg, "Superradiance effect from bunches of relativistic electron-oscillators," Sov. Tech. Phys. Lett., vol. 14, no. 4, pp. 197-201, 1988.

[12] N. S. Ginzburg and A. S. Sergeev, "Superradiance in layers of excited quantum and nonisochronous classical oscillators," Opt. Cоттип., vol. 91, nos. 1-2, pp. 140-146, Jul. 1992.

[13] N. S. Ginzburg, I. V. Zotova, A. S. Sergeev, I. V. Konoplev, A. D. R. Phelps, A. W. Cross, S. J. Cooke, V. G. Shpak, M. I. Yalandin, S. A. Shunailov, and M. R. Ulmaskulov, "Experimental observation of cyclotron superradiance under group synchronism conditions," Phys. Rev. Lett., vol. 78, no. 12, pp. 2365-2368, Mar. 1997.

[14] N. S. Ginzburg, N. Y. Peskov, I. V. Zotova, A. S. Sergeev, A. D. R. Phelps, A. W. Cross, W. He, K. Ronald, V. G. Shpak, M. I. Yalandin, S. A. Shunailov, and M. R. Ulmaskulov, "Experimental observation of wiggler superradiance under group synchronism condition," Nucl. Instrum. Meth. Phys. Res. A, Accel., Spectrometers, Detectors Assoc. Equip., vol. 429, nos. 1-3, pp. 94-100, Jun. 1999.

[15] N. S. Ginzburg, A. S. Sergeev, Y. V. Novozhilova, I. V. Zotova, R. M. Rozenthal, A. D. R. Phelps, A. W. Cross, P. Aitken, V. G. Shpak, M. I. Yalandin, S. A. Shunailov, and M. R. Ulmaskulov, "Experimental observation of Cherenkov superradiance from an intense electron bunch," Opt. Commun., vol. 175, nos. 1-3, pp. 139-146, Feb. 2000.

[16] A. G. Reutova, M. R. Ul'maskulov, A. K. Sharypov, V. G. Shpak, S. A. Shunailov, M. I. Yalandin, V. I. Belousov, N. S. Ginzburg, G. G. Denisov, I. V. Zotova, R. M. Rozental', and A. S. Sergeev, "Experimental observation of superradiance effect in the process of backscattering of powerful microwave pump wave by high-current relativistic electron bunch with subnanosecond duration," JETP Lett., vol. 82, no. 5, p. 263, 2005.

[17] N. S. Ginzburg, A. S. Sergeev, I. V. Zotova, Y. V. Novozhilova, N. Y. Peskov, I. V. Konoplev, A. D. R. Phelps, A. W. Cross, S. J. Cooke, P. Aitken, V. G. Shpak, M. I. Yalandin, S. A. Shunailov, and M. R. Ulmaskulov, "Experimental observation of superradiance in millimeter-wave band," Nucl. Instrum. Meth. Phys. Res. A, vol. 393, nos. 1-3, pp. 352-355, Jul. 1997.

[18] N. S. Ginzburg, N. Y. Novozhilova, I. V. Zotova, A. S. Sergeev, N. Y. Peskov, A. D. R. Phelps, S. M. Wiggins, A. W. Cross, K. Ronald, W. He, V. G. Shpak, M. I. Yalandin, S. A. Shunailov, M. R. Ulmaskulov, and V. P. Tarakanov, "Generation of powerful subnanosecond microwave pulses by intense electron bunches moving in a periodic backward wave structure in the superradiant regime," Phys. Rev. E, vol. 60, no. 3, pp. 3297-3304, 1999.

[19] M. I. Yalandin, V. G. Shpak, S. A. Shunailov, M. R. Oulmaskoulov, N. S. Ginzburg, I. V. Zotova, Y. V. Novozhilova, A. S. Sergeev, A. D. R. Phelps, A. W. Cross, S. M. Wiggins, and K. Ronald, "Generation of powerful subnanosecond microwave pulses in the range of 38-150 GHz," IEEE Trans. Plasma Sci., vol. 28, no. 5, pp. 1615-1619, Oct. 2000. 
[20] S. D. Korovin, G. A. Mesyats, V. V. Rostov, M. R. Ulmaskulov, K. A. Sharypov, V. G. Shpak, S. A. Shunailov, and M. I. Yalandin, "High effective generation of subnanosecond microwave pulses in the relativistic BWO," Tech. Phys. Lett., vol. 28, no. 1, pp. 76-81, Jan. 2002.

[21] A. A. Eltchaninov, S. D. Korovin, V. V. Rostov, I. V. Pegel, G. A. Mesyats, S. N. Rukin, V. G. Shpak, M. I. Yalandin, and N. S. Ginzburg, "Production of short microwave pulses with a peak power exceeding the driving electron beam power," Laser Particle Beams, vol. 21, no. 2, pp. 187-196, Aug. 2003.

[22] S. D. Korovin, G. A. Mesyats, V. V. Rostov, M. R. Ulmaskulov, K. A. Sharypov, V. G. Shpak, S. A. Shunailov, and M. I. Yalandin, "Subnanosecond source of microwave pulses of $38 \mathrm{GHz}$ with peak power of 1 GW," Tech. Phys. Lett., vol. 30, no. 3, p. 68, 2004.

[23] D. M. Grishin, V. P. Gubanov, S. D. Korovin, S. K. Lubutin, G. A. Mesyats, A. V. Nikofirov, V. V. Rostov, S. N. Rukin, B. G. Slovikovsky, K. A. Sharypov, V. G. Shpak, S. A. Shunailov, and M. I. Yalandin, "Generation of powerful subnanosecond microwave pulses of $38 \mathrm{GHz}$ with repetition frequency $3500 \mathrm{~Hz}$," Pis'ma v ZhTF, vol. 28, no. 19, pp. 24-31, 2002.

[24] S. K. Luybutin, S. N. Rukin, K. A. Sharypov, V. G. Shpak, S. A. Shunailov, B. G. Slovikovsky, M. R. Ulmaskulov, M. I. Yalandin, S. D. Korovin, and V. V. Rostov, "Nanosecond hybrid modulator for the fast-repetitive driving of X-band, gigawatt-power microwave source," IEEE Trans. Plasma Sci., vol. 33, no. 4, pp. 1220-1225, Apr. 2005.

[25] S. D. Korovin, A. A. Eltchaninov, V. V. Rostov, V. G. Shpak, M. I. Yalandin, N. S. Ginzburg, A. S. Sergeev, and I. V. Zotova, "Generation of Cherenkov superradiance pulses with a peak power exceeding the power of driving short electron beam," Phys. Rev. E, vol. 74, no. 1, pp. 016501-1-016501-8, Jul. 2006

[26] K. V. Afanas'ev, N. M. Bykov, V. P. Gubanov, A. A. El'chaninov, A. I. Klimov, S. D. Korovin, V. V. Rostov, and A. S. Stepchenko, "A high-power periodic nanosecond pulse source of coherent 8cm electromagnetic radiation," Tech. Phys. Lett., vol. 32, no. 11, pp. 925-927, Nov. 2006.

[27] D. M. Grishin, S. K. Lyubutin, G. A. Mesyats, V. V. Rostov, S. N. Rukin, B. G. Slovikovsky, S. P. Timoshenkov, M. R. Ulmaskulov, K. A. Sharypov, V. G. Shpak, S. A. Shunailov, and M. I. Yalandin, "Generation of gigawatt 10-GHz pulses with stable phase," Tech. Phys. Lett., vol. 34, no. 10, pp. 822-824, Oct. 2008.

[28] M. I. Yalandin, A. G. Reutova, M. R. Ulmaskulov, K. A. Sharypov, V. G. Shpak, S. A. Shunailov, A. I. Klimov, V. V. Rostov, and G. A. Mesyats, "Picosecond stability of injection of parallel high-current pulsed electron beams," Tech. Phys. Lett., vol. 35, no. 9, pp. 804-807, Sep. 2009

[29] A. A. El'chaninov, A. I. Klimov, O. B. Koval'chuk, G. A. Mesyats, I. V. Pegel', I. V. Romanchenko, V. V. Rostov, K. A. Sharypov, and M. I. Yalandin, "Coherent summation of power of nanosecond relativistic microwave generators," Tech. Phys., vol. 56, no. 1, pp. 121-126, 2011.

[30] V. V. Rostov, A. A. Elchaninov, I. V. Romanchenko, and M. I. Yalandin, "A coherent two-channel source of Cherenkov superradiance pulses,' Appl. Phys. Lett., vol. 100, no. 22, pp. 224102-1-224102-4, May 2012.

[31] V. V. Rostov, M. I. Yalandin, and G. A. Mesyats, "Repetitive production of nanosecond gigawatt microwave pulses," IEEE Trans. Plasma Sci., vol. 36, no. 3, pp. 655-660, Jun. 2008.

[32] M. I. Yalandin, S. A. Shunailov, M. R. Ul'maskulov, K. A. Sharypov, V. G. Shpak, V. V. Rostov, I. V. Romanchenko, A. A. El'chaninov, and A. I. Klimov, "Synphase operation of nanosecond relativistic 37-GHz backward-wave oscillators without electrodynamic coupling," Tech. Phys. Lett., vol. 38, no. 10, p. 917-920, Oct. 2012.

[33] V. R. Baryshev, N. S. Ginzburg, I. V. Zotova, A. S. Sergeev, R. M. Rozental, and M. I. Yalandin, "Amplification of ultrashort electromagnetic pulses propagating along quasistationary electron beams," Tech. Phys., vol. 79, no. 1, pp. 103-109, Jan. 2009.

[34] M. I. Yalandin, A. G. Reutova, M. R. Ul'maskulov, K. A. Sharypov, S. A. Shunailov, N. S. Ginzburg, I. V. Zotova, E. R. Kocharovskaya, and A. S. Sergeev, "Effect of the nonlinear compression of ultrashort microwave pulses in the process of the amplification by quasistationary electron beams, J. Experim. Theoretical Phys. Lett., vol. 91, no. 11, pp. 553-557, Aug. 2010.

[35] N. S. Ginzburg, A. S. Sergeev, and I. V. Zotova, "Self-induced transparency and electromagnetic pulse compression in a plasma or an electron beam under cyclotron resonance conditions," Phys. Rev. Lett., vol. 105 , no. 26 , pp. 265001-1-265001-4, Dec. 2010.
[36] N. S. Ginzburg, I. V. Zotova, and A. S. Sergeev, "Self-induced transparency, compression, and stopping of electromagnetic pulses interacting with beams of unexcited classical oscillators," J. Experim. Theoretical Phys., vol. 113, no. 5, pp. 772-780, Nov. 2011.

[37] N. S. Ginzburg, S. P. Kuznetsov, and T. N. Fedoseeva, "Theory of transit processes in relativistic BWT," Radiofizika, vol. 21 , no. 7, pp. 1037-1052, 1978

[38] G. S. Nusinovich, Introduction to the Physics of Gyrotons. Baltimore, MD, USA: The Johns Hopkins Univ. Press, 2004.

[39] S. E. Harris, "Electromagnetically induced transparency," Phys. Today, vol. 50, no. 7, pp. 36-42, Jul. 1997.

[40] L. V. Hau, S. E. Harris, Z. Dutton, and C. H. Behrrozi, "Light speed reduction to 17 metres per second in an ultracold atomic gas," Nature, vol. 397, no. 6720, pp. 594-598, Feb. 1999.

[41] D. F. Phillips, A. Fleischhauer, A. Mair, R. L. Walsworth, and M. D. Lukin, "Storage of light in atomic vapors," Phys. Rev. Lett., vol. 86, no. 5, pp. 783-786, 2001.

[42] S. G. Tantawi, R. D. Ruth, A. E. Vlieks, and M. Zolotorev, "Active high-power RF pulse compression using optically switched resonant delay lines," IEEE Trans. Microw. Theory Tech., vol. 45, no. 8, pp. 1486-1492, Aug. 1997.

[43] S. V. Samsonov, A. D. R. Phelps, V. L. Bratman, G. Burt, G. G. Denisov, A. W. Cross, K. Ronald, W. He, and H. Yin, "Compression of frequencymodulated pulses using helically corrugated waveguides and its potential for generating multigigawatt RF radiation," Phys. Rev. Lett., vol. 92, no. 11, pp. 118301-1-118301-4, Mar. 2004.

[44] J. G. Power, "Overview of photoinjectors," in Proc. 14th Adv. Accel. Concepts Workshop, Jun. 2010, pp. 20-28.

[45] P. Piot, Y. Sun, and K. Kim, "Photoinjector generation of a flat electron beam with transverse emittance ratio of 100," Phys. Rev. ST Accel. Beams, vol. 9, no. 3, pp. 031001-1-031001-7, Mar. 2006.

[46] N. S. Ginzburg, A. M. Malkin, A. S. Sergeev, and V. Y. Zaslavsky, "Quasi-optical theory of relativistic submillimeter surface-wave oscillators," Appl. Phys. Lett., vol. 99, no. 12, pp. 121505-1-121505-3, Sep. 2011.

[47] B. Z. Katsenelenbaum, "Irregular waveguides with slowly changed parameters," Dokl. Akad. Nauk SSSR, vol. 102, pp. 711-714, May 1961.

[48] S. J. Smith and E. M. Purcell, "Visible light from localized surface charges moving across a grating," Phys. Rev., vol. 92, no. 4, p. 1069 , Nov. 1953.

[49] A. S. Kesar, "Smith-purcell radiation from a charge moving above a finite-length grating," Phys. Rev. ST Accel. Beams, vol. 8, no. 7, pp. 072801-1-072801-7, Jul. 2005.

[50] S. E. Korbly, A. S. Kesar, J. R. Sirigiri, and R. J. Temkin, "Observation of frequency-locked coherent terahertz smith-purcell radiation," Phys. Rev. Lett., vol. 94, no. 5, pp. 054803-1-054803-4, Feb. 2005.

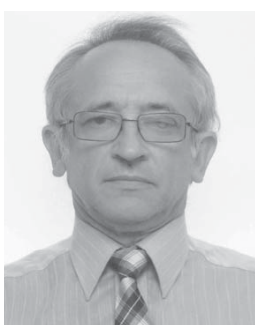

Naum S. Ginzburg was born in 1952. He received the M.Sc. degree in physics from Gorky State University, Kharkiv, Ukraine, in 1974, and the Ph.D. and Doctor of Science degrees from the Institute of Applied Physics, Russian Academy of Science, Nizhny Novgorod, Russia, in 1983 and 1994, respectively.

$\mathrm{He}$ has been a Professor with the Institute of Applied Physics since 2002. He is currently the Head of the Group of Theory of Relativistic Microwave Devices, Institute of Applied Physics. His current research interests include high-power microwave electronics, including free electron laser, cyclotron resonance masers, gyrotrons, scattering electromagnetic waves by relativistic electron beams, as well as optical guiding and superradiance.

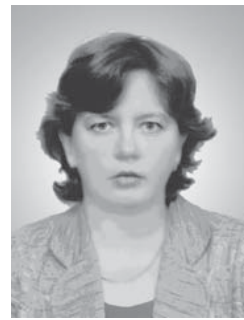

Irina V. Zotova was born in 1968. She received the M.Sc. degree in physics from Gorky State University, Kharkiv, Ukraine, in 1990, and the Ph.D. degree from the Institute of Applied Physics, Russian Academy of Science, Nizhny Novgorod, Russia, in 1999. She is currently a Senior Research Scientist with the Institute of Applied Physics.

Her current research interests include gyrotrons, relativistic electronics, optical guiding, and superradiance of classical electron bunches. 


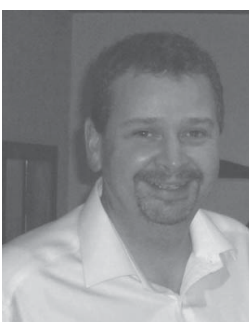

Adrian W. Cross received the B.Sc. degree (Hons.) in physics and the Ph.D. degree from the University of Strathclyde, Glasgow, U.K., in 1989 and 1993, respectively.

He joined the Atoms, Beams, and Plasmas Group, University of Strathclyde, in 1993, initially as a Research Fellow and then as a Lecturer in 2000 , Senior Lecturer in 2005, and a Reader in 2007 with the Department of Physics. From 2002 to 2007, he was an Engineering and Physical Science Advanced Fellow. He has been involved in various aspects of research on gyrotrons, cyclotron autoresonance masers, free-electron lasers, superradiant sources, gyrotron traveling wave amplifiers and plasma applications. His current research interests include $\mathrm{THz}$ radiation sources and pseudospark physics.

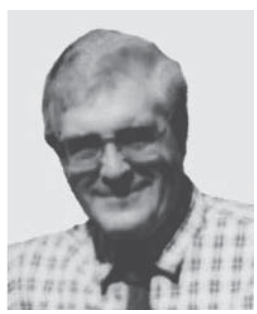

Alan D. R. Phelps (M'07) received the B.A. degree (Hons.) in physics and the M.A. degree from Cambridge University, Cambridge, U.K., in 1966 and 1970 , respectively, and the D.Phil. degree in plasma research from Oxford University, Oxford, U.K., in 1970.

He has been with the University of Strathclyde, Glasgow, U.K., since 1978, becoming a full Professor in 1993 and the Head of the Physics Department from 1998 to 2001. His current research interests include high-power free-electron radiation sources and plasmas.

Dr. Phelps is a fellow of the American Physical Society, the UK Institute of Physics, and the Royal Society of Edinburgh.

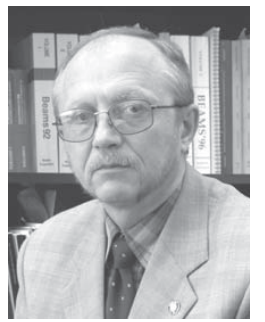

Michael I. Yalandin (M’09) was born in 1956. He received the M.Sc. degree in plasma physics from Novosibirsk State University, Novosibirsk, Russia, in 1979, the Candidate Sci. and Doctor Sci. degrees from the Institute of High Current Electronics, Russian Academy of Science, Tomsk, Russia, in 1985 and 1996, respectively.

He is currently the Head of the Group with the Institute of Electrophysics, Russian Academy of Science, which is currently engaged in the investigation and development of compact high-voltage nanosecond and subnanosecond modulators, pulsed electron accelerators and high-power sources of coherent and broadband microwaves.

Dr. Yalandin was the recipient of the Komsomol Award in science in 1987, and the State Award of Russian Federation in science in 1998. He was elected Corresponding Member of the Russian Academy of Science in 2003.

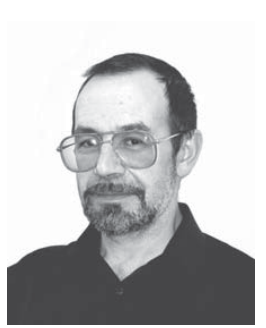

Vladislav V. Rostov received the M.Sc. degree in plasma physics from Novosibirsk State University, Novosibirsk, Russia, in 1978, the Candidate Sci. and Doctor Sci. degrees from the Institute of High Current Electronics, Russian Academy of Science, Tomsk, Russia, in 1985 and 2001, respectively.

$\mathrm{He}$ has been with the Institute of High Current Electronics since 1978. He is currently a Leader of a research group dealing with theoretical investigations in high-power microwave electronics.

Dr. Rostov was the recipient of the Komsomol Award in science in 1987 and the State Award of Russian Federation in science in 2003. 